\title{
Optimisation of Design and Operation of Generators for Offshore Vertical Axis Wind Turbines
}

\author{
Michael Argent ${ }^{1}$, Alasdair McDonald ${ }^{1}$, William Leithead ${ }^{1}$, Alexander Giles ${ }^{1}$ \\ 1 Wind Energy \& Control Centre, Electrical and Electronic Engineering, University of Strathclyde, \\ Glasgow, United Kingdom
}

\begin{abstract}
A process for optimising both the design and operation of the generator for a large offshore vertical axis wind turbine (VAWT) is developed. The objectives of the optimisation process are to minimise additional costs and losses in the generator to allow for a fair evaluation of the impact of the VAWT environment on the powertrain. A spectrum of torque control strategies was tested based on the ratio, $q$, of the allowed electrical torque variation to the inherent mechanical torque variation. Equations relating $q$ to the generator losses were established. The effect of $q$ on the energy extracted by the rotor was also investigated and incorporated into the optimisation process. This work shows that a variable $q$ strategy with respect to wind speed can improve turbine performance across the range of operational wind speeds depending on the torque loading from the rotor blades. In turn, this also allows for the torque rating of the generator to be reduced from the peak torque rating that would otherwise be expected, creating an opportunity to downscale the generator size, reducing costs. The optimisation of powertrain design and operation should be carried out at as high level as is possible, ideally using the fully factored Cost of Energy (COE) to guard against unexpected losses due to excessive focus in one $\mathrm{COE}$ factor (for example reducing upfront cost but in turn reducing availability).
\end{abstract}

\section{KEYWORDS}

vertical axis wind turbine; drivetrain; optimisation; permanent magnet generator; direct drive; wind

\section{Correspondence}

M. Argent, Wind \& Marine Energy Systems Centre for Doctoral Training, Electrical and Electronic Engineering, University of Strathclyde, Glasgow, United Kingdom

E-mail: michael.argent@strath.ac.uk

\section{Acknowledgements}

The work demonstrated in this paper has been funded by the Engineering and Physical Sciences Research Council (EPSRC) in the United Kingdom (project number EP/G037728/1) and has been supported by the University of Strathclyde. The authors would also like to acknowledge Prof Michael Graham (Imperial College) and Dr Carlos Simao Ferreira (TU Delft) for their contribution to the Panel method code used to model the torque generated from the VAWT rotor blades.

\section{List of Symbols}

$A_{\mathrm{e}} \quad$ Iron Loss Eddy Current Loss Coefficient

$A_{\mathrm{h}} \quad$ Iron Loss Hysteresis Loss Coefficient

$\hat{B}_{F e} \quad$ Iron Flux Density

$\hat{B}_{0} \quad$ Baseline Iron Flux Density $=1.5 \mathrm{~T}^{10}$

$b \quad$ Number of rotor blades

$C_{\mathrm{p}} \quad$ Power Coefficient

$E_{\mathrm{p}} \quad$ Induced emf

$\mathcal{F}_{m} \quad$ Magnet MMF 


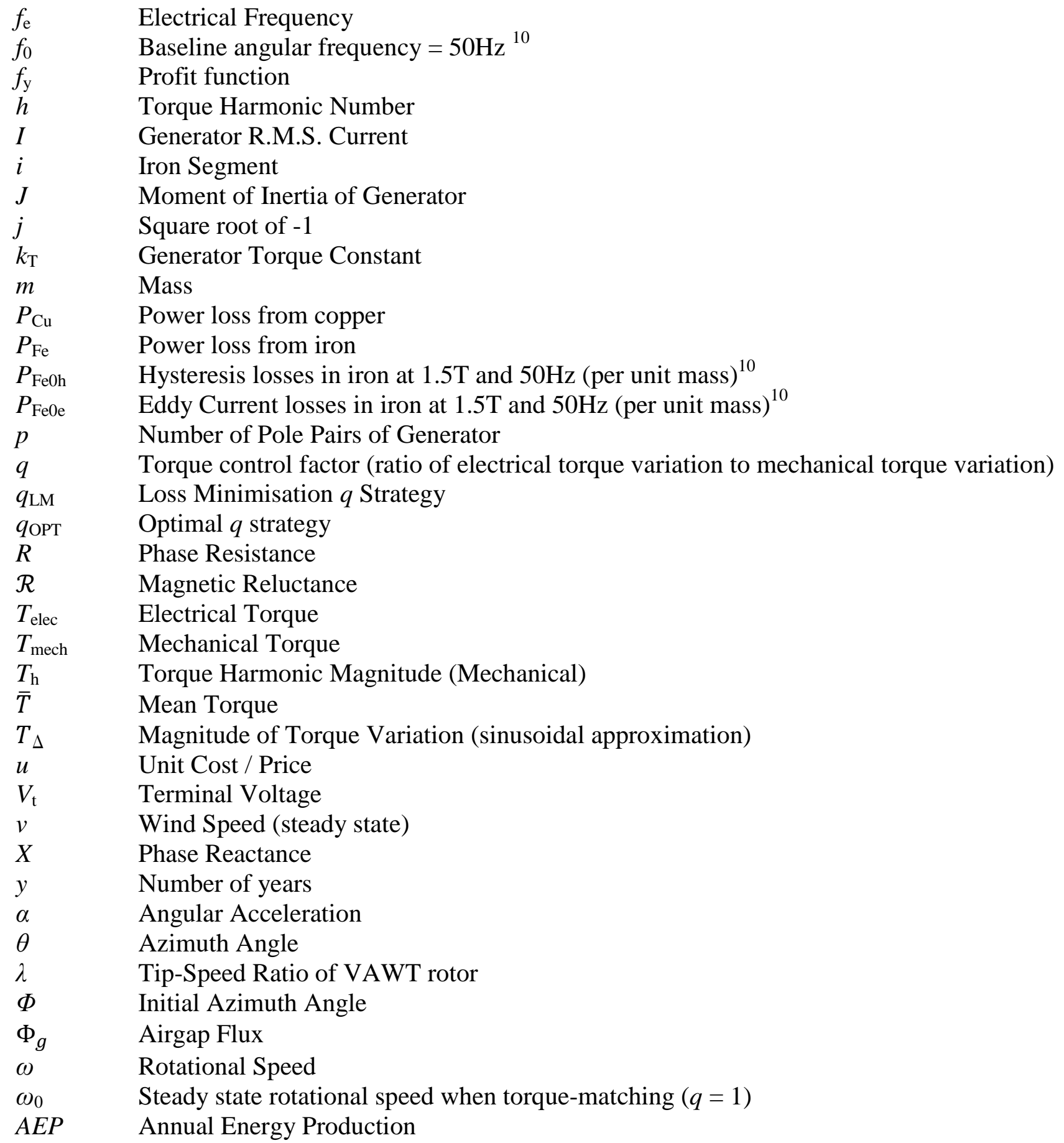

\section{INTRODUCTION}

This work proposes new torque control strategies for generators of VAWTs and demonstrates how these strategies influence the costs and efficiency of the powertrain and energy capture of the VAWT. It uses a nested optimisation process on both the powertrain design and operation and demonstrates that to minimise cost of energy, these torque control strategies should vary with wind speed which in addition allows the powertrain to be rated for a lower torque value than the peak mechanical torque. 


\subsection{Offshore VAWTs}

There is increasing interest in the use of Vertical Axis Wind Turbines (VAWTs) for large-scale deployment in the offshore environment. Notable academic and commercial VAWT projects include the Sandia National Laboratories, ${ }^{1}$ the NOVA project's Aerogenerator V-rotor, ${ }^{2}$ the INFLOW project's Nenuphar Twinfloat concept, ${ }^{3}$ the VertAx H-rotor, ${ }^{4}$ the VertiWind $2 \mathrm{MW}$ prototype, ${ }^{5}$ and the Sea Twirl offshore demonstrator. ${ }^{6}$

Compared to their Horizontal axis counterparts, VAWTs do not require yaw systems, do not experience gravitational cyclic loading, and some designs reduce or eliminate the tower. Some researchers have claimed that the turbine blades for VAWTs can be longer than the equivalent Horizontal Axis Wind Turbines (HAWTs), reducing the mass and cost of larger (e.g. 10-20MW) offshore wind turbines. ${ }^{7}$ With the potential additional benefits of accessing powertrain and control equipment at or near sea level, there is scope for improving O\&M costs too. These benefits might mean a lower Cost of Energy (COE) overall for VAWTs.

Unfortunately, VAWTs also have a number of challenges compared to HAWTs, driven by the aerodynamics inherent to a VAWT which impact on the design and control of the powertrain. Typically VAWTs operate at lower tip-speed ratios than HAWTs. This reduced rotor speed results in an increased torque rating for the same power output increasing powertrain costs and losses. In addition, since the angle of attack and apparent wind speed experienced by a blade vary during each revolution, strongly cyclic forces arise even at constant wind speeds. As the rotor rotates, there is also an interaction between the blades and the downstream wake and this leads to a cyclic nature of the torque input to the powertrain, i.e. a peak rotor torque which can be substantially greater than the mean torque. This too can lead to further increases in powertrain cost.

Before a powertrain can be tailored to a given VAWT design, one must decide whether the powertrain is designed for peak rotor torque or the mean rotor torque at the rated wind speed. It is this question - and the various implications of the answer on the powertrain design - that this paper investigates.

As with some offshore HAWTs, the trend for prototype multi-MW VAWTs is towards a directdrive powertrain. For example, the VertAx design consists of two 5MW inverted PMGs mounted on the outside of the tower connecting to the blades by the upper and lower rotor hubs respectively. ${ }^{4}$

This paper examines the evaluation of direct-drive generators for large VAWTs by modelling the equivalent magnetic and electric circuits of the generator to determine the losses and costs associated with different generator designs and with different operational strategies. A key contribution of this paper is the definition of a variable which describes how much the electrical torque of the generator matches the mechanical torque. The paper develops models to see how this variable then affects the electrical losses in the generator at different wind speeds by simulating the torque loading on the generator generated by the rotor blades and then calculating the equivalent magnetic and electric circuit for the generator to calculate power output and losses. This process summarised in Figure 1. 


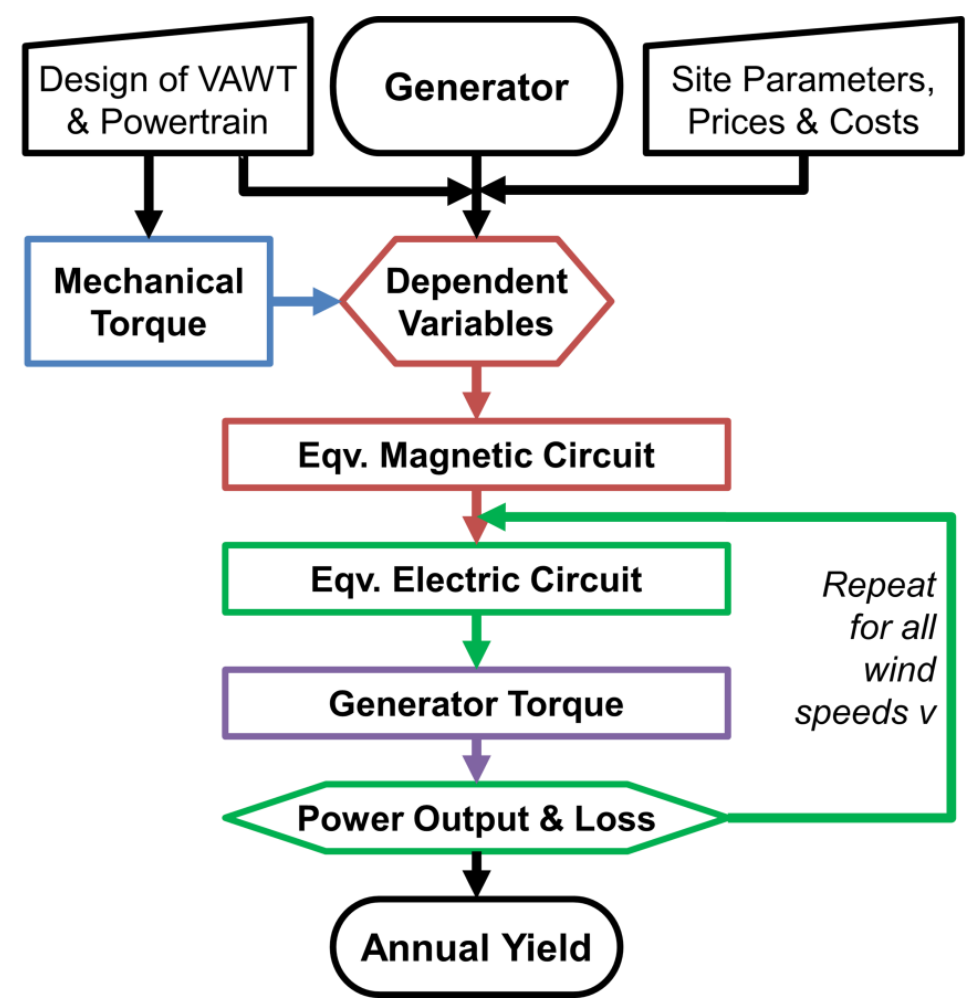

Figure 1. Overview of VAWT Generator Modelling Process

\subsection{Torque Harmonics and Torque Control}

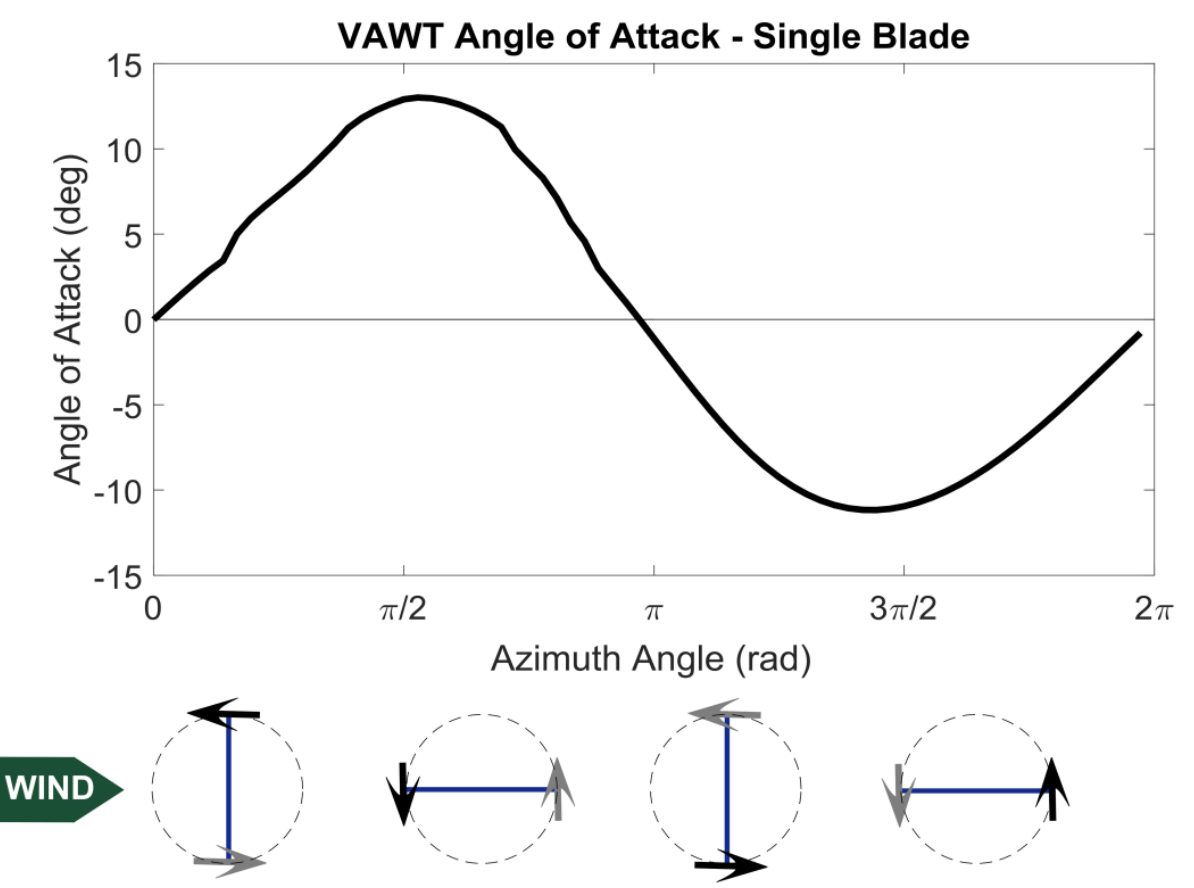

Figure 2. Geometric Angle of Attack for a VAWT blade against Azimuth Angle

Figure 2 shows how the angle of attack for a VAWT rotor blade varies throughout each rotor revolution (at a given mean rotor speed) as the apparent wind angle changes. This is also dependent on the tip-speed ratio. ${ }^{9}$ This leads to a varying resultant force generated by the blades. When summed up for all the blades, this produces a cyclic torque pattern. 
The resulting torque has significant periodicity which can be characterised using Fourier analysis. In order to explore the development of a methodology, this cyclic mechanical torque $T_{\text {mech }}$ is initially modelled as a mean torque $\bar{T}$ with a fundamental periodic component of magnitude $T_{\Delta}$ and a frequency equal to the rotor speed $\omega$ multiplied by the number of blades (which in this case is $b=2$ ) that varies with azimuth angle $\theta$. This is shown in equation (1),

$$
T_{\text {mech }} \cong \bar{T}+T_{\Delta} \sin (b \theta+\Phi)=\bar{T}+T_{\Delta} \sin (b \omega t+\Phi)
$$

where $\Phi$ indicates the initial angular position. In a HAWT, in steady-state conditions, the opposing electrical torque $T_{\text {elec }}$ developed by the generator is constant. If that constant generator torque was translated to the VAWT, the cyclic mechanical rotor torque would produce an angular acceleration. The response of the rotating inertia to a torque imbalance is defined by equation (2),

$$
T_{\text {mech }}-T_{\text {elec }}=J \alpha
$$

where $J$ is the combined moment of inertia of the wind turbine's rotor, any shafts and the directdrive generator's rotor, and $\alpha$ is the angular acceleration of the rotor caused by the torque imbalance. This will result in a variation in rotational speed $\omega$ about a mean rotor speed. Another option is that the electrical torque (which is controlled by adjusting the generator current) matches the mechanical torque at every azimuth angle. In this case, there is no angular acceleration; that is, the rotor speed is constant. Between these two extremes of operating strategy, the generator torque can match only some of the mechanical torque variation. Assuming that $\bar{T}_{\text {elec }}=\bar{T}_{\text {mech }}$ (i.e. mean torques are equal so that the rotor speed only varies within a rotation, rather than from one rotation to another) a torque factor, $q$ can be defined as the ratio of the electrical torque variation to the mechanical torque variation (as described by equation (3))

$$
q=\frac{T_{\Delta_{\text {elec }}}}{T_{\Delta_{\text {mech }}}}
$$

The electrical torque profiles and the resulting change in rotor speed for different $q$ strategies are demonstrated in Figure 3, where $q=0$ shows the constant generator torque $\bar{T}$, varying rotational speed case and $q=1$ is the case where the generator torque follows the mechanical torque exactly leading to a constant rotational speed (and a peak torque of $\bar{T}+T_{\Delta}$ ). If $0<q<1$ then this implies variation in both electrical torque and rotational speed (Figure 2 shows the case when $q=0.5$ ).

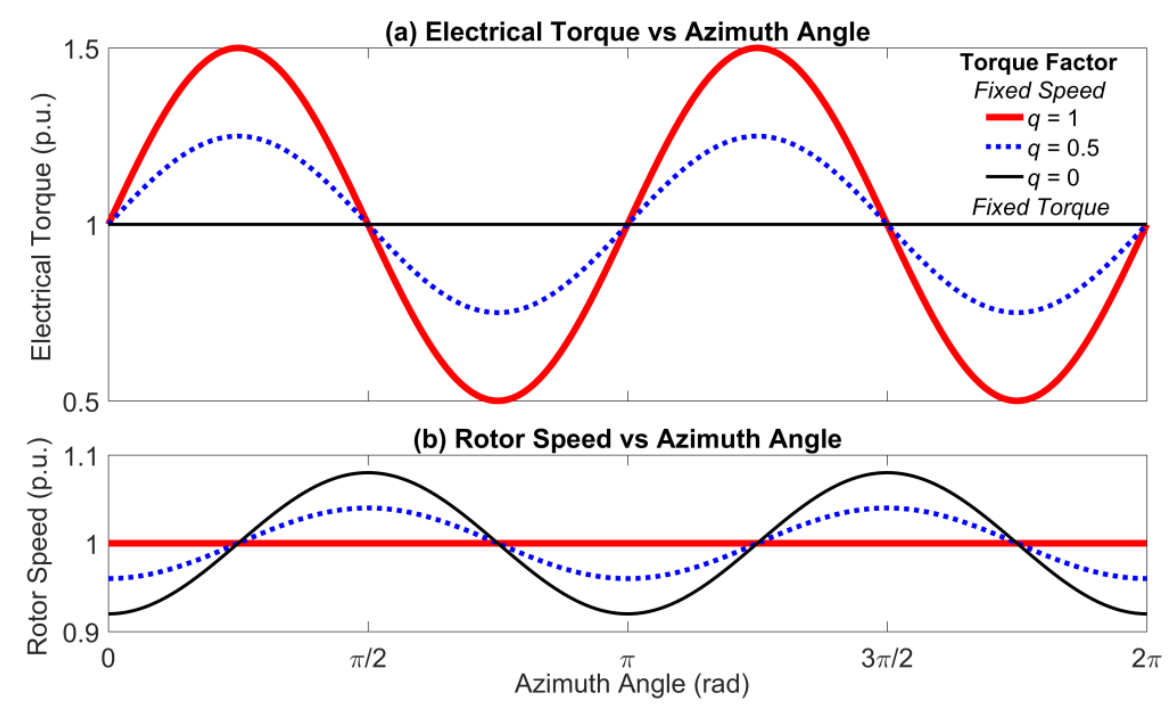

Figure 3. Cyclic variation of (a) Electrical torque, (b) Rotor speed for different $q$ factors 
This can be further developed for all harmonics $h$, and not just $h=b$. An illustration of the actual torque profile and the corresponding harmonic components are shown in Figure 4. Equation (1) can be extended to all torque harmonics, leading to equation (4) where $T_{\mathrm{h}}$ represents the magnitude of mechanical torque harmonic (replacing $T_{\Delta}$ ). Equation (5) shows the electrical torque, where the concept of $q$ is applied to each individual harmonic, $q_{\mathrm{h}}$. In this research it is assumed that $q$ is applied proportionally to each harmonic, i.e. $q_{1}=q_{2}=q_{3}=\ldots=q_{\mathrm{n}}=q$ as demonstrated by the dotted line in Figure $4 \mathrm{a}$ where $q=0.5$ is applied to all harmonics (compared with the solid $q=1$ waveform and the flat $q=0$ strategy).

$$
\begin{gathered}
T_{\text {mech }}=\bar{T}+\sum_{h=1}^{n} T_{h} \sin \left(h \theta+\Phi_{h}\right)=\bar{T}+\sum_{h=1}^{n} T_{h} \sin \left(h \omega t+\Phi_{h}\right) \\
T_{\text {elec }}=\bar{T}+q \sum_{h=1}^{n} T_{h} \sin \left(h \theta+\Phi_{h}\right)=\bar{T}+q \sum_{h=1}^{n} T_{h} \sin \left(h \omega t+\Phi_{h}\right)
\end{gathered}
$$

(a) Electrical Torque Response

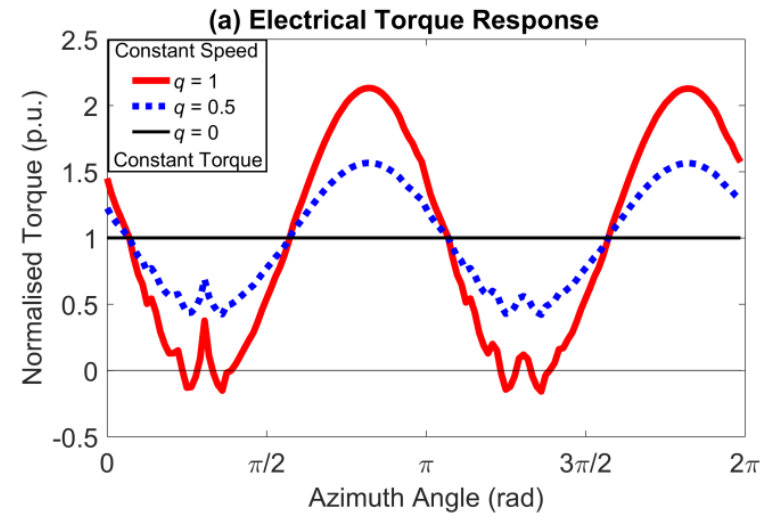

(b) Harmonics of Cyclic Mechanical Torque

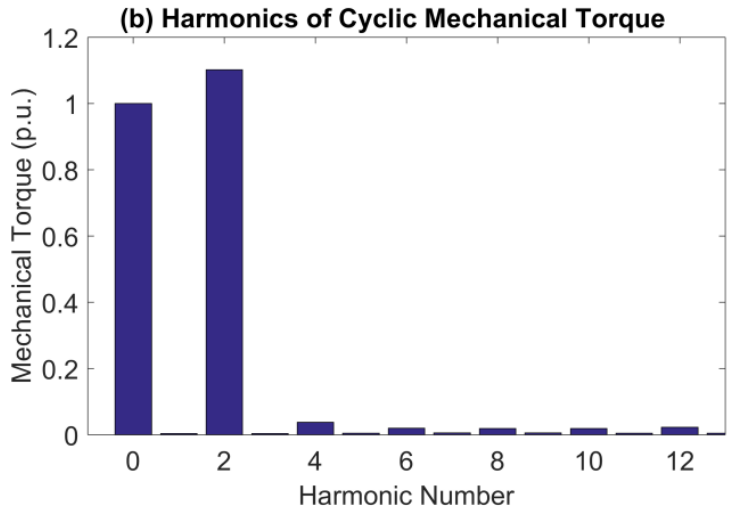

Figure 4. (a) Cyclic Electrical Torque Variation, (b) Cyclic Torque Harmonic Components

In section 2, the effect of $q$ on torque- and speed-related powertrain losses will be evaluated by developing quasi-steady-state analytical expressions for the copper and iron losses. In section 3 this paper touches on the aerodynamic consequences of these operational strategies and the resulting effect on the energy extraction. Finally, section 4 will introduce an optimisation approach for both control strategies and generator design in order to maximise the energy production while limiting generator costs.

There are a number of different types of VAWT, as well several different top-level system design variables that have a major impact on the characteristics and performance of the VAWT as well as influencing the optimal powertrain design. In this paper, the baseline VAWT is a 10MW, 2 bladed H-rotor with a medium swept area aspect ratio (i.e. the ratio of blade length and blade separation). The powertrain is a direct-drive permanent magnet generator. Table I shows the range of some of the VAWT design parameters (including power rating and rotor design) that can be specified when designing a VAWT and associated powertrain. The values highlighted in bold are the parameters selected for the baseline VAWT and powertrain which most of the analysis in this paper is conducted on. In addition, section 5.2 tests the effects of two design variables: swept area aspect ratio and turbine rating (highlighted in grey) on powertrain optimisation. 


\section{GENERATOR MODELLING}

The VAWTs investigated here use a direct drive permanent magnet generator. Polinder et al. suggested a steady-state model of the generator which can be used to evaluate performance at multiple wind speed. ${ }^{10}$ Defined in terms of a number of independent variables, this type of model gives iron and copper losses and can be used for optimisation. The generator steady-state loss models will be developed for torque and rotational speed varying within a rotational cycle. The resulting loss and its variation with respect to $q$ will be presented.

\subsection{Generator Magnetic Circuit}
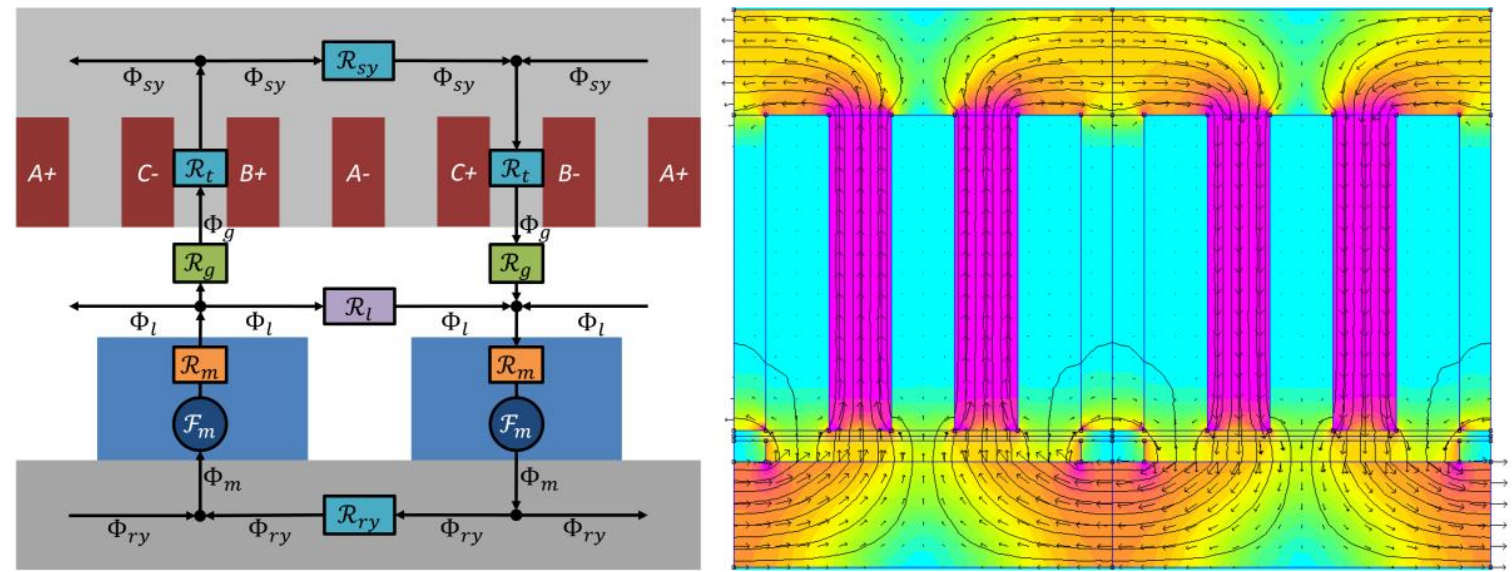

Figure 5. (a) Magnetic Circuit Illustration, (b) Magnetic Flux Density modelled in FEMM

Figure 5a illustrates the magnetic circuit model comprising a single pole pair segment of the generator. The model starts with a lumped parameter magnetic circuit to find the flux for each pole. Ampere's, Gauss's and Hopkinson's Laws can be used to find the airgap flux $\Phi_{g}$ in terms of the magnet MMF $\mathcal{F}_{m}$ (proportional to magnet height) and reluctances $\mathcal{R}$ around the magnetic circuit. This is formulated in Equation (6).

$$
\Phi_{g}=\frac{\mathcal{F}_{m}}{\left(\mathcal{R}_{\mathrm{m}}\left(1+\frac{4 \mathcal{R}_{g}}{\mathcal{R}_{l}}\right)+\mathcal{R}_{\mathrm{g}}\right)}
$$

This flux per pole can then be used to find the fundamental airgap flux density using Fourier analysis of a square wave which then allows density allows the induced EMF to be calculated for every wind speed value. The airgap flux densities were verified cross referencing a Finite Element Analysis model constructed in the open access package FEMM as shown in Figure 5 b. 


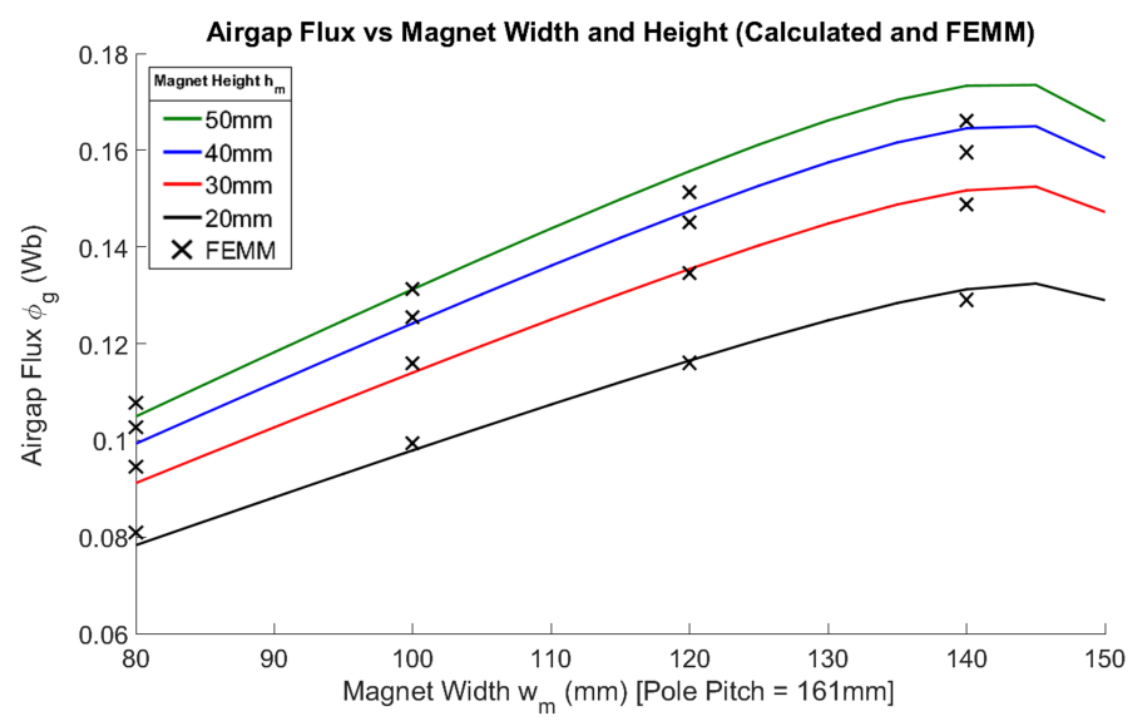

Figure 6. Airgap Flux vs Magnet Width and Height (Calculated and FEMM)

Figure 6 illustrates how the airgap flux varies with the magnet width and height in both models. This shows that the magnetic circuit model and the FEMM model are in close agreement up until a magnet width $95 \%$ of pole pitch which justifies using the arithmetic approach for modelling the multiple generator configurations required in the optimisation process.

\subsection{Generator Electrical Model}

For a given tip speed ratio, each wind speed value corresponds to one value of rotational speed. Since the rotational speed varies with azimuth angle when $0 \leq q<1$, both the induced EMF and electrical frequency will also vary around the azimuth for a given wind speed.

An equivalent electrical circuit is then used to model the terminal voltage for each wind speed and rotor position. The varying electrical frequency means that the reactance also varies with azimuth angle (except when $q=1$ ). For a given design, the electrical torque, $T_{\text {elec }}$, developed by the generator can be modelled as described by equation (7):

$$
T_{\text {elec }}=k_{T} I
$$

where $k_{\mathrm{T}}$ is the generator's torque constant and $I$ is the root-mean square (RMS) current in the generator windings. Since the electric torque is harmonic, the RMS current is also harmonic, modelled as a sum of a mean RMS current $\bar{I}$ and a harmonic series of sine components with magnitude of $q I_{h}$ (where $I_{h}$ corresponds to the mechanical torque harmonic $T_{h}$ ) varying with azimuth angle and $q$ :

$$
I=\frac{\bar{T}}{k_{T}}+\frac{q}{k_{T}} \sum_{h=1}^{n} T_{h} \sin \left(h \theta+\Phi_{h}\right)
$$

If $q=0$ (i.e. fixed torque for a given wind speed) then the current is constant for a fixed wind speed; if $q=1$ (i.e. electrical torque tracks mechanical torque) then the magnitude of variation in current will be at its largest. The terminal voltage $V_{\mathrm{t}}$ then depends on (potentially) variable induced emf $E_{\mathrm{p}}$, the phase reactance $X$, the phase resistance $R$, and the phase current $I$ determined by the relationship in Equation (9) derived from the phasor diagram of a generator assumed to be operating at unity power factor.

$$
V_{\mathrm{t}}=E_{\mathrm{p}}-j I X-I R_{\mathrm{s}}
$$


These electrical circuit parameters are used to calculate the power output of the generator as well as generator losses, notably copper and iron losses described in the next section.

\subsection{Copper and iron losses and $q$}

The major loss types in a permanent magnet generator are the stator copper losses and the stator iron losses. The copper losses depend on the current through the stator coils which is proportional to the electrical torque response. The copper losses $P_{\mathrm{Cu}}$ are calculated by averaging the $I^{2} R$ losses over one complete revolution of the rotor (by integrating the harmonic form of $I$ (Equation (8)) across the entire revolution to determine the mean $I^{2}$ value). The resulting form is as shown in Equation (10) demonstrating that the increase in copper losses varies with the square of $q$ and of the square of the current harmonics $I_{h}$ (where the copper losses are at their maximum by selecting the $q=1$ fixed speed strategy),

$$
P_{\mathrm{Cu}}=R\left(\bar{I}^{2}+\frac{q^{2}}{2} \sum_{h=1}^{n} I_{h}{ }^{2}\right)
$$

The iron losses are related to the electrical frequency, that is the product of the number of pole pairs $p$ and the rotational speed of the rotor. This rotational speed is calculated by first combining equations (2) and (11) to rewrite $\omega$ in terms of the torque imbalance and then torque harmonics (equations (4) and (5)). This is then integrated over one complete revolution to calculate the mean values of both the electrical frequency, given by equation (12), and the square of the electrical frequency, given by equation (13).

$$
\begin{gathered}
\omega^{2}=\omega_{0}^{2}+2 \alpha \theta \\
\bar{f}_{\mathrm{e}}=\frac{p}{2 \pi}\left(\omega_{0}+\frac{1}{J \omega_{0}}\left(q_{h}-1\right) \sum_{h=1}^{n} \frac{T_{h}}{h}\right) \\
\overline{f_{\mathrm{e}}^{2}}=\left(\frac{p}{2 \pi}\right)^{2}\left(\omega_{0}^{2}+\frac{2}{J}\left(q_{h}-1\right) \sum_{h=1}^{n} \frac{T_{h}}{h}\right)
\end{gathered}
$$

The equation for calculating iron losses is derived from equation (14) in Polinder's paper, ${ }^{10}$ calculating the specific losses (per unit mass) for each steel segment $i$ individually (dependent on both $\bar{f}_{\mathrm{e}}$ and $\overline{f_{\mathrm{e}}^{2}}$ in equations (12) and (13)), then multiplied by their respective masses $m_{\mathrm{i}}$ and summed to give the form in equation (14) below. For clarity certain iron constants were grouped into the coefficients $A_{\mathrm{h}}$ representing hysteresis losses, and $A_{\mathrm{e}}$ representing eddy current losses (where $P_{\mathrm{Fe} 0 \mathrm{~h}}$ and $P_{\mathrm{Fe} 0 \mathrm{e}}$ are the specific type losses per unit mass at defined frequency $f_{0}$ and flux density $\hat{B}_{0}{ }^{2}$, while $\hat{B}_{\mathrm{Fe}}$ is the amplitude of flux density in the specific segment).

$$
P_{\mathrm{Fe}}=\sum_{i}\left(A_{\mathrm{h}} \overline{f_{\mathrm{e}}}+A_{\mathrm{e}} \overline{f_{\mathrm{e}}{ }^{2}}\right) \hat{B}_{\mathrm{Fe}_{i}}{ }^{2} m_{i} \text { where } A_{\mathrm{h}}=\frac{2 P_{\mathrm{Fe} 0 \mathrm{~h}}}{f_{0} \hat{B}_{0}{ }^{2}} ; A_{\mathrm{e}}=\frac{2 P_{\mathrm{Fe} 0 \mathrm{e}}}{{f_{0}{ }^{2} \hat{B}_{0}{ }^{2}}^{2}}
$$

The iron losses have a component that is proportional to $(q-1)$; therefore, the iron losses are largest at fixed speed operation (where $q=1$ ) and are minimised at fixed torque operation (where $q=0$ ). Note that these iron losses, as with the copper losses, are average power losses across each revolution.

As for the relative importance of the torque harmonics on generator losses, the copper losses depend on the magnitude of $T_{h}^{2}$. This means that only the largest magnitudes of $T_{\mathrm{h}}$ have a significant impact. On the other hand, the iron losses depend on $T_{\mathrm{h}} / h$ meaning that the higher harmonics will have less of an impact. For 2 bladed VAWTs, since the $2^{\text {nd }}$ harmonic is dominant (e.g. Figure $3 b$ ), it is likely that the large majority of the copper and iron losses will come from the simple sum of the 
fixed component and the $2^{\text {nd }}$ harmonic (i.e. the other harmonics contribute only a small part of the generator losses).

Figure 7 shows how the generator losses vary with $q$ when subjected to harmonic torque input (as shown in Figure 3a). Since the torque variation is large in these tests $\left(T_{\Delta}=1.1 \bar{T}\right)$, there is a large peak torque experienced which, due to the $I^{2}$ relationship of the copper losses, means that there is a large range in copper losses. The strategy that minimises generator losses is the fixed torque $q=0$ strategy, with the fixed speed strategy $(q=1)$ suffering from a large increase in copper losses. Also of note is that the majority of losses are explained by the fixed component and the $2^{\text {nd }}$ harmonic (for a two bladed VAWT); thus, this simple sum provides a good estimate of total generator losses. ${ }^{12}$
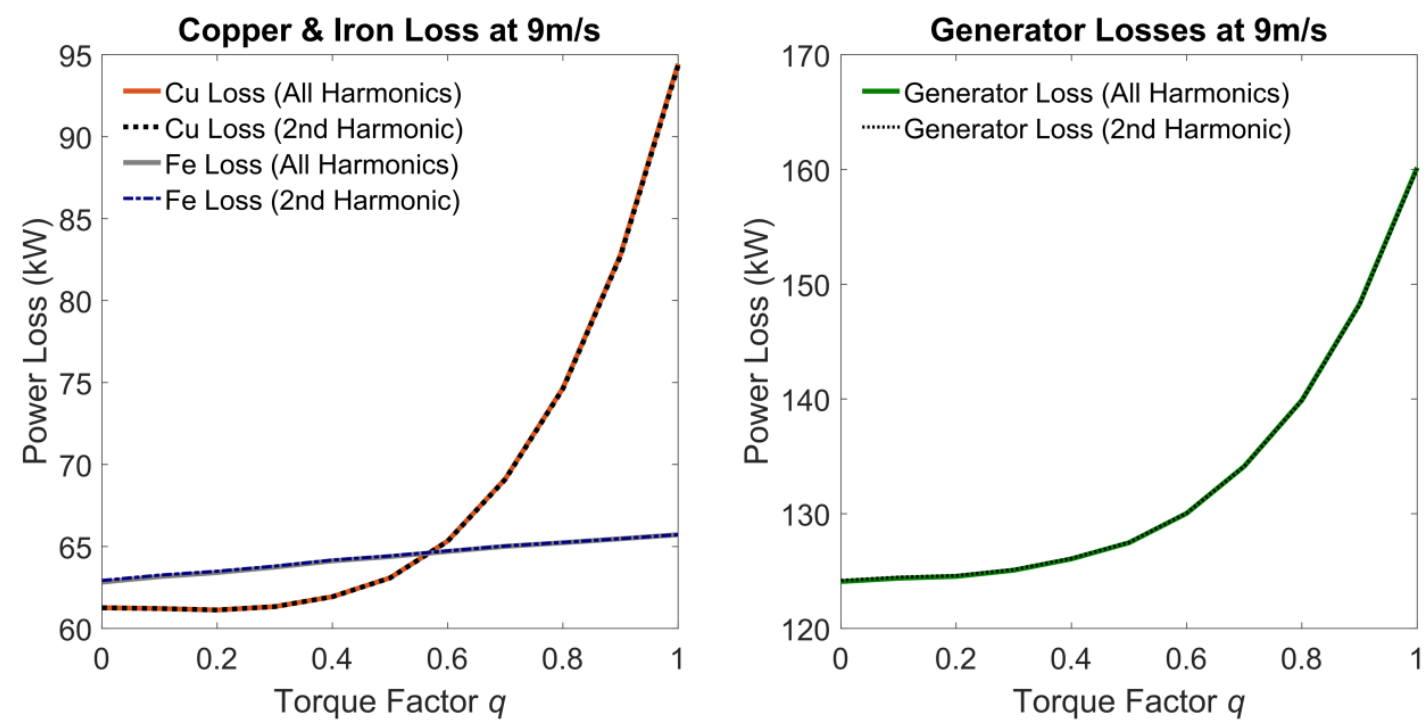

Figure 7. Generator Losses at $7 \mathrm{~m} / \mathrm{s}$ with Harmonic Torque

Figure 8 shows how generator losses compare for different $q$ strategies across the range of wind speeds (compared relative to the $q=0$ fixed torque baseline). The strategy to minimise losses $q_{\mathrm{LM}}=0$ for all speeds (i.e. fixed torque). This plot shows the $q_{\mathrm{OPT}}$ strategy (elaborated on in sections 4 and 5) that optimises the torque control strategy to maximise the generator's power output for each wind speed. In general this $q_{\mathrm{OPT}}$ strategy employs a higher $q$ at low wind speeds and a lower $q$ at high wind speeds where copper losses have a great effect. 

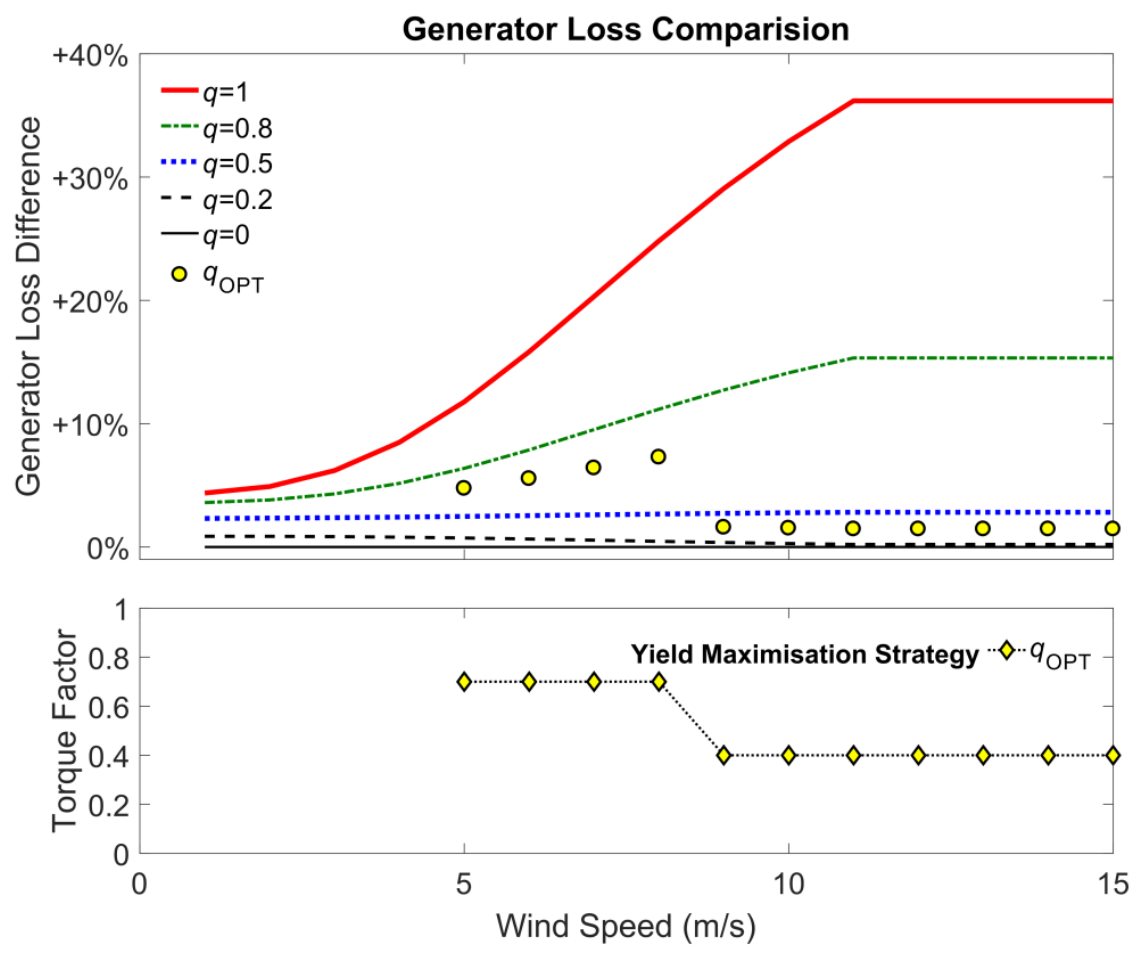

Figure 8. Generator Losses vs $q$ for all wind speeds (and $q_{\mathrm{OPT}}$ strategy)

\subsection{Peak Electrical Torque Loading}

The peak electrical torque that the generator can develop is an important factor in the design and cost of the generator. Figure 9 shows how this is affected by $q$ setting and wind speed, with the peak torque experienced at rated operation. The lower $q$ is, the lower peak electrical torque is. Of note is the loss minimisation $q_{\mathrm{OPT}}$ strategy which has a relatively low peak electrical torque (since $q_{\mathrm{OPT}}$ is near zero at rated). Additionally, the peak electrical torque rating for $q=1$ depends on whether all harmonics or just the $2^{\text {nd }}$ torque harmonic are taken into account. ${ }^{12}$

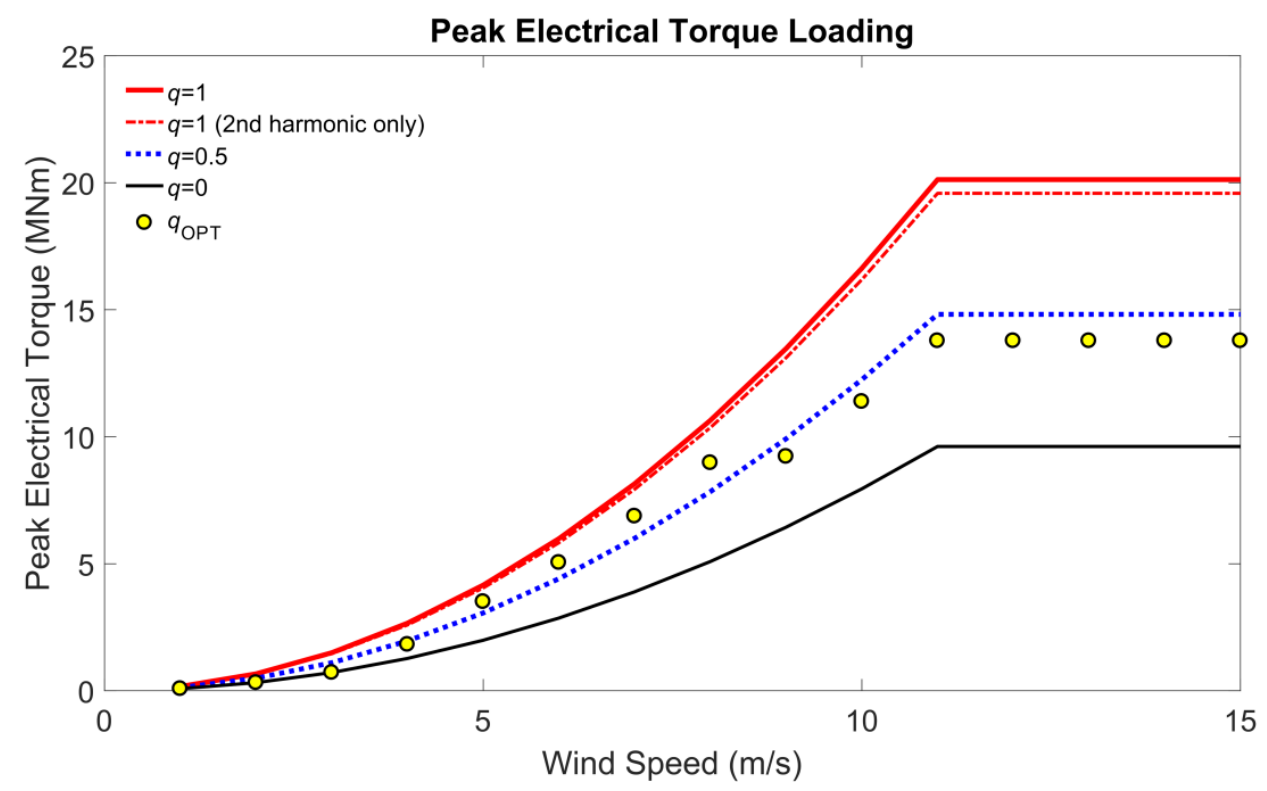

Figure 9. Peak Electrical Torque Loading for different $q$ strategies. 


\section{AERODYNAMIC MODELLING}

Section 2 introduced generator loss models as a function of $q$. It is anticipated that varying rotational speed with rotor angle will influence the energy extraction of the rotor. With the exception of the fixed speed $q=1$ strategy, the rotor speed will be a function of azimuth. This will introduce an additional degree of variation to both the angle of attack and apparent wind speed experienced by the blades. Accordingly, the aerodynamics should not be modelled using an algorithm which applies averaging in some form or another to variables such as the rotor speed; instead a time-stepping algorithm is required. Since this method is significantly slower than the rest of the optimisation process, it is easier to separately consider the modelling of the aerodynamically induced mechanical torque and its resultant effect on the generator (including power generated and losses).

\subsection{Aerodynamic vortex code}

Multiple methods for modelling the aerodynamics of VAWTs have been developed for various research activities. In the 1980s, the preferred algorithm was the Double Multiple Streamtube (DMST) algorithm, which is an algorithm based on the Blade Element Momentum (BEM) theory. This models the incoming airflow into a series of streamtubes which have energy absorbed by actuator "discs"; for DMST codes, there are two actuator "discs" one upwind and one downwind of the VAWT rotor for each streamtube. ${ }^{13}$ However, the DMST algorithm assumes a constant rotor speed for a fixed wind speed which is incompatible with the methodology of this research.

At the other extreme are Computational Fluid Dynamics (CFD) techniques which numerically solve the Navier-Stokes equations of the fluid interaction with the turbine. While they have the ability to analyse complex aerodynamic phenomena including flow separation when the blades are stalled, the computational time required for these models is significant (especially when running multiple variations for different $q$ settings). ${ }^{13}$

Given that the focus of this work is on the evaluation and optimisation of the VAWT powertrain, it was decided that a vortex code that follows the approach of Ferreira ${ }^{14}$ would be a more appropriate modelling technique. This algorithm models the wake as an incompressible and irrotational vortex system, with induction factors calculated by summing up velocities induced by all the vortex segments in the wake. The bulk of the computational cost is in evaluating the wake's self-influence at the end of a time-step; however, through parallelisation the cost of this section can be kept to reasonable levels. Embedded into vortex codes are Kelvin's circulation theorem, which stipulates that the rate of change of circulation in a closed system is zero, and the Kutta condition, which stipulates that the trailing edge of a blade is a stagnation point (as shown in figure 10). Kelvin's theorem is of particular importance for dynamic systems. ${ }^{14-15}$

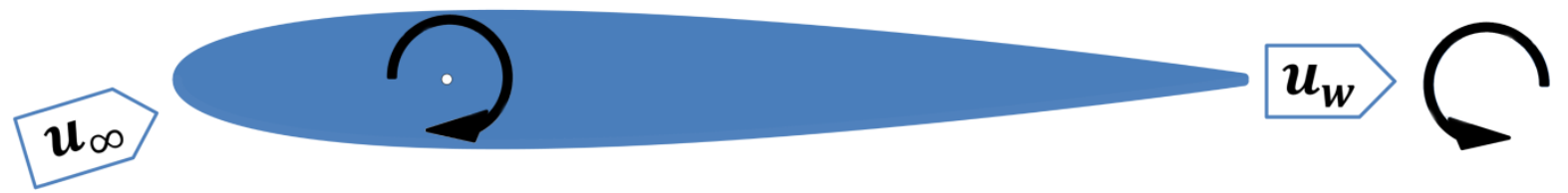

Figure 10. Circulation around a blade

The time-stepping nature of this algorithm's implementation allows for its use in situations where the rotor speed varies with time. Previous work by Ferreira and Zanon suggests that such a technique is adequate for determining the apparent wind speed experienced by the blades along with the angle of attack and induction factors at high Reynolds numbers and tip-speed ratios $\lambda>3 .{ }^{16}$ 
Since the code does not model dynamic stall, it can be used as long as the rotational speed is not allowed to drop too low. ${ }^{22}$

\subsection{Implementation to calculate changes in energy extraction due to $q$ and $\lambda$}

To model the effect of this dynamic wake on the input torque to the generator, the rotational speed at each time step must be known. The change in rotor speed is calculated (Equation (2)) from the torque balance between the mechanical torque (from all rotor blades) and electrical torque dependent on $q$. This new rotor speed is then used in the calculation of the aerodynamic loading at the next time step. Once the simulation has been completed, the resulting relationship of mechanical torque to azimuth angle is established and the harmonic components extracted using Fourier analysis, which are then passed on to the generator loss equations as well as for calculating the mean mechanical power for each $q$ calculated by averaging the power generated from the product mechanical torque of the VAWT rotor and its rotational speed. This process is repeated for a number of combinations of wind speed and torque factor, $q$.

A reduction in the mean rotor mechanical power output for different $q$ strategies can be considered as a third source of power loss (in addition to copper and iron losses). This loss is defined as the difference between the largest mean mechanical power (for all $q$ cases) and the mean mechanical power for a particular $q$ case. This effect can be analysed by using the vortex code to calculate the mechanical torque and rotational speed for each wind speed $v$ and torque factor $q$ combination and then working through the generator code to calculate the mean mechanical power output (across the whole azimuth range) for each combination of $v$ and $q$. Once this is completed, the largest mean mechanical power can be found for each wind speed.

The effect of mean tip speed ratio can also be examined using this method. This is done by changing different mean rotational speeds, $\bar{\omega}$, and repeating the process described above. Different H-rotor designs have different $\overline{C_{\mathrm{p}}}-\bar{\lambda}$ characteristics depending on their design (where $\overline{C_{\mathrm{p}}}$ refers to the output power coefficient and $\bar{\lambda}$ is the tip speed ratios with each parameter averaged over one revolution). ${ }^{17}$ The change in this mean rotational speed $\bar{\omega}$ leads to a change in mean value of tip speed ratio and hence of power coefficient. It is likely that the optimal $q$ strategy will be somewhat dependent on this mean tip speed ratio.

\subsection{Aerodynamic Loss Results}
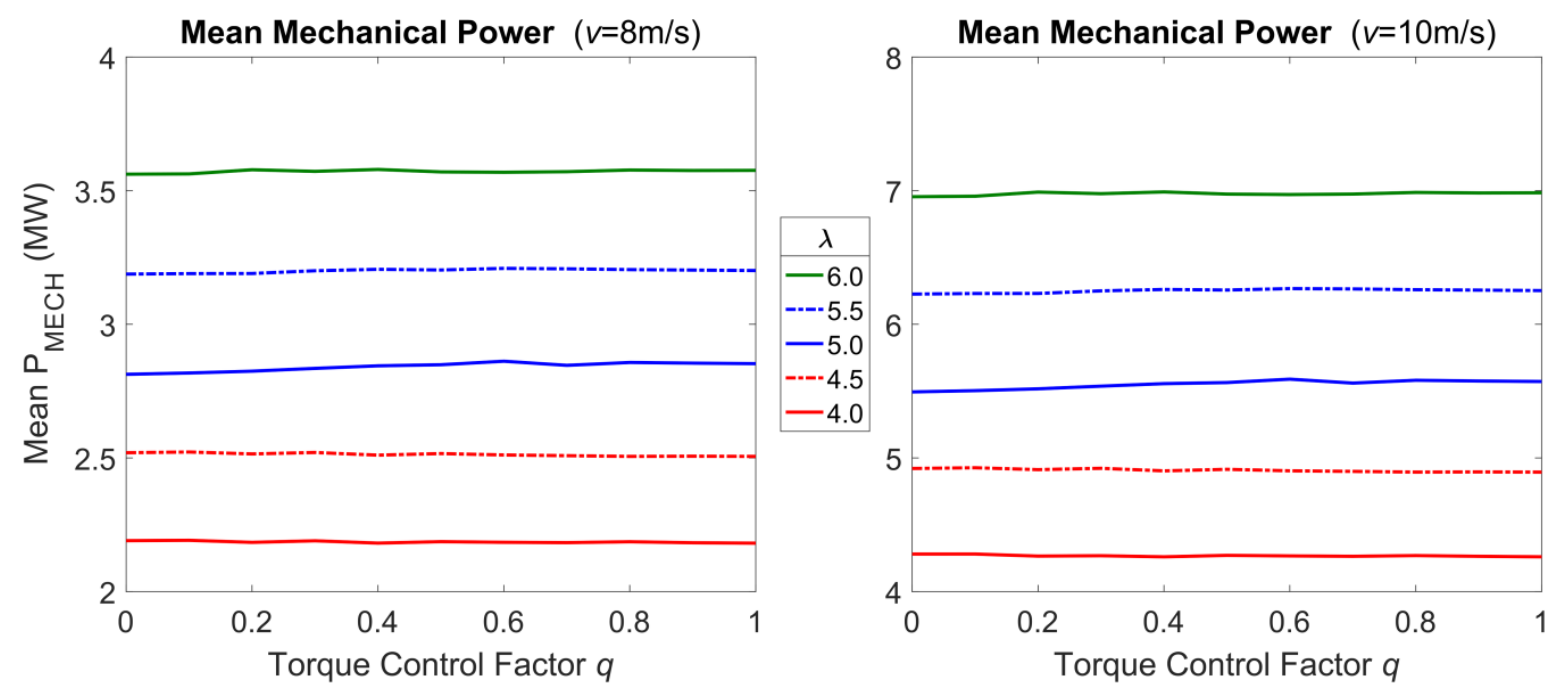

Figure 11. Mean Mechanical Power Output at (a) $8 \mathrm{~m} / \mathrm{s}$, (b) $10 \mathrm{~m} / \mathrm{s}$ 
Figure 11 shows the average mechanical power output and its variation with torque factor, $q$, for mean tip speed ratios $\bar{\lambda}$ between 4 and 6 , at wind speeds of $8 \mathrm{~m} / \mathrm{s}$ and $10 \mathrm{~m} / \mathrm{s}$. The focus on this work was on the below rated regime where the efficiency of the generator has a greater impact on the energy production of the turbine. There is an increase in mean mechanical power output as $\bar{\lambda}$ increases. There is also some small variation in how $q$ affects mechanical power depending on $\bar{\lambda}$. It is small in magnitude and its relationship with $q$ is difficult to quantify but even a small increase in mechanical power with $q$ can affect the optimal strategy to maximise power. Figure 12a shows how angle of attack decreases with $\bar{\lambda}$ which corresponds to the increasing $\overline{C_{\mathrm{p}}}-\bar{\lambda}$ curve in Figure $12 \mathrm{~b}$.

The vortex code used in this model does not include drag components, in practice the VAWT's $\overline{C_{\mathrm{p}}}-\bar{\lambda}$ curve would peak at a relatively low tip-speed ratio. However this does not affect the methodology of this research, nor that the main focus is on the powertrain response of the VAWT.
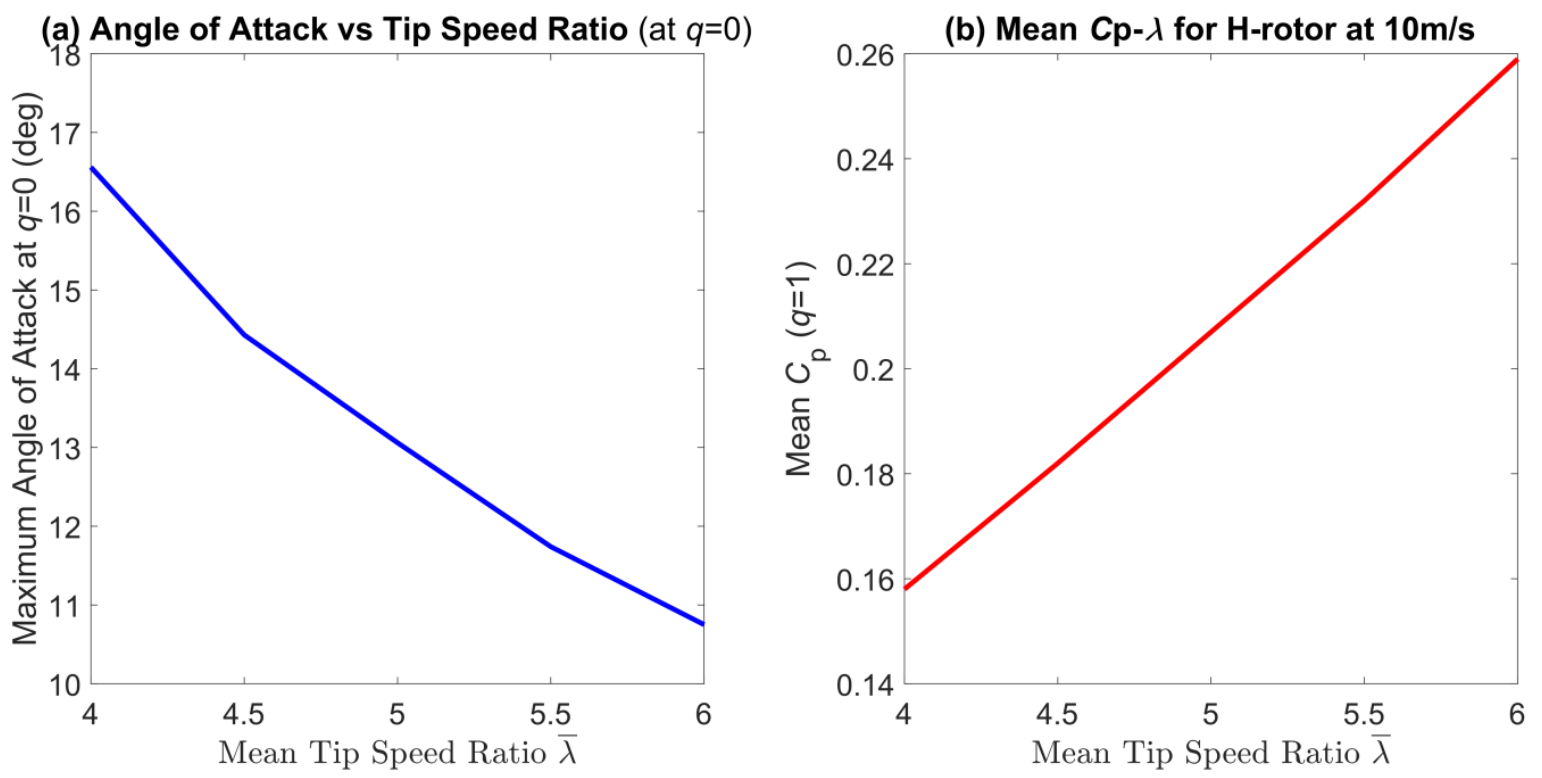

Figure 12. (a) Maximum Angle of Attack, (b) Power Coefficient vs Tip Speed Ratio $\left(\overline{\boldsymbol{C}_{\mathbf{p}}}-\overline{\boldsymbol{\lambda}}\right)$

\section{GENERATOR OPTIMISATION}

In order to optimise the cost of energy of the VAWT powertrain, this paper proposes a nested optimisation routine, taking into account the specifics of the VAWT rotor design, in particular the cyclic torque pattern. An inner control strategy loop seeks to find the maximum energy production by varying $q$ at each wind speed for a given generator design; while the outer machine design loop that seeks to find the optimal design of the generator by varying a number of generator design parameters.

The overall optimisation process is shown in Figure 13. The aerodynamic model is run before the optimisation based on a range of predefined discrete combinations of wind speed, mean tip speed ratio, and $q$ typically for a large number of rotations until the mean mechanical torque converges and mean rotational speed converges. The Fourier coefficients of the resulting mechanical torque and speed data is stored in a data file and can be called up for a number of different generator designs.

The main part of the program is the generator design optimisation loop which determines the combination of generator parameters (e.g. magnet width and height, slot width and height, airgap, and so on) that minimises the objective function (see section 4.1.1) and is thus determined to be the optimal generator design. This outer optimisation loop includes a generator model calculating the equivalent magnetic circuit and the equivalent electric circuit for the given generator design in an 
iteration. This is passed into the inner generator control optimisation loop which runs through the different control strategies and computes the effect of the cyclic torque on the power output and associated losses for each strategy and determines the optimal control strategy $q_{\mathrm{OPT}}(v)$ for this generator design which is defined as the $q$ setting that maximises the power output for each wind speed $v$. This is then used to calculate the annual energy yield (using the Weibull distribution) and the resulting objective function value for the outer optimisation.

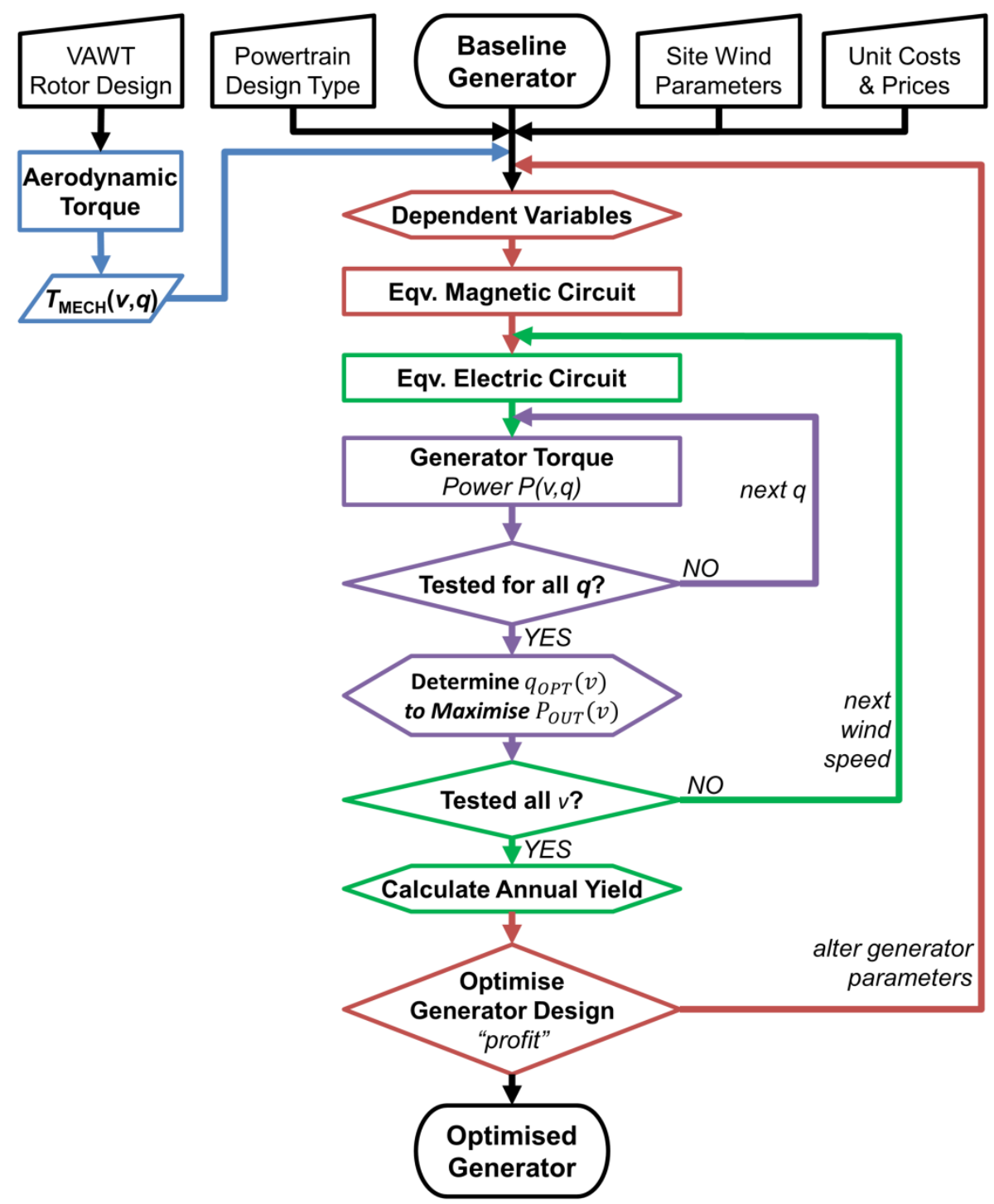

Figure 13. Top Level Algorithm for Generator Optimisation Program

\subsection{Generator Design Optimisation}

This section describes the objective function and the optimisation algorithm.

\subsubsection{Objective Function}

The ideal objective function for many design processes in wind energy is the cost of energy. This requires a large number of parameters that are outside the powertrain design that are difficult to estimate (e.g. the cost of the turbine rotor, foundation costs) and which have a large uncertainty. It has been shown in that a good proxy for cost of energy in powertrain optimisation is an objective function which tries to minimise the powertrain cost while maximising the annual energy yield. ${ }^{18}$ It 
has been assumed for this study that this result for HAWTs is equally applicable to VAWTs. The objective function used here is shown in Equation (15) where the generator active cost is the total cost of the electrical steel $(€ 3 / \mathrm{kg})$, copper $(€ 15 / \mathrm{kg})$ and permanent magnets $(€ 25 / \mathrm{kg})$ used in the generator (unit prices $u$ as used by Polinder) ${ }^{10}$ and the revenue is the energy yield over $y$ years (either 5,10 or 15 ) multiplied by an assumed value of electricity (e.g. $u=€ 190 / \mathrm{MWh}$ ).

$$
\begin{aligned}
& \text { Objective Function = Generator Active Cost }- \text { Revenue } \\
& f_{y}=\left(u_{C u} m_{C u}+u_{F e} m_{F e}+u_{P M} m_{P M}\right)-\left(u_{M W h} \times y \times A E P\right)
\end{aligned}
$$

\subsubsection{Independent Variables}

The implementation of the complete algorithm (Figure 13) involved carrying out a five-parameter optimisation of the generator designed for use in a 10MW offshore VAWT with an annual mean wind speed of $9.8 \mathrm{~m} / \mathrm{s}$ (following a Weibull distribution with the same scaling parameters as Hart, et.al.). ${ }^{19}$

Table II shows the 7 parameters that are the independent variables for the generator design optimisation (outer loop) process (in contrast to the inner loop optimisation where the only independent variable is $q$ for each wind speed) along with the corresponding lower and upper bounds (to be used in all upcoming tests) as well as the default value (used in the pre-optimised baseline case) for each variable.

The choices of independent variables determine a series of dependent variables. For instance, the pole pitch is fixed based on the stator radius and the integer number of pole pairs, while the magnet width depends on the magnet width ratio and the pole pitch. The airgap clearance, $g$, is taken to be the $0.1 \%$ of the stator diameter, a standard rule of thumb used in the design of larger radius generators. ${ }^{19}$

\subsubsection{Genetic Algorithm \& Pattern Search}

The optimisation algorithm used in this program is a hybrid approach of the Genetic Algorithm and Pattern Search techniques. ${ }^{18}$ These algorithms take in a range of independent variables, any constraints and minimise the given objective function within this range of variables.

The Genetic Algorithm mimics the process of natural selection that drives biological evolution. It creates a population of candidate solutions (thereby running the generator calculation code multiple times within a single generation) and each generation uses the results of these evaluations ("parents") to evolve a new population of candidate solutions ("children") using selection (carrying forward the best performing parents), crossover (combining elements from a pair of parents) and mutation (random changes to a single parent). ${ }^{20}$ After multiple generations the population approaches the optimal area, although it can take a long time to converge to the optima. Therefore, after a small number of iterations the output from the GA is used as the input for another algorithm to quickly pinpoint the optima.

The Pattern Search algorithm searches a set of points ("mesh") around the current point looking for one that improves on the previous best value of objective function (i.e. if changing any of the design variables improve Cost of Energy). This process continues: narrowing the mesh when it finds no improvement and expanding the mesh out from any new best point when discovered, ultimately converging on the global minima (i.e. the optimal generator configuration). ${ }^{21}$

This hybrid optimisation algorithm is described by the flow chart in Figure 14 and was used by McDonald and Bhuiyan for optimising PM generators for HAWTs. ${ }^{18}$ Tests involving three variables (magnet width, magnet height and airgap) takes around three minutes on a standard desktop PC to run in full from start to optimal solution. 


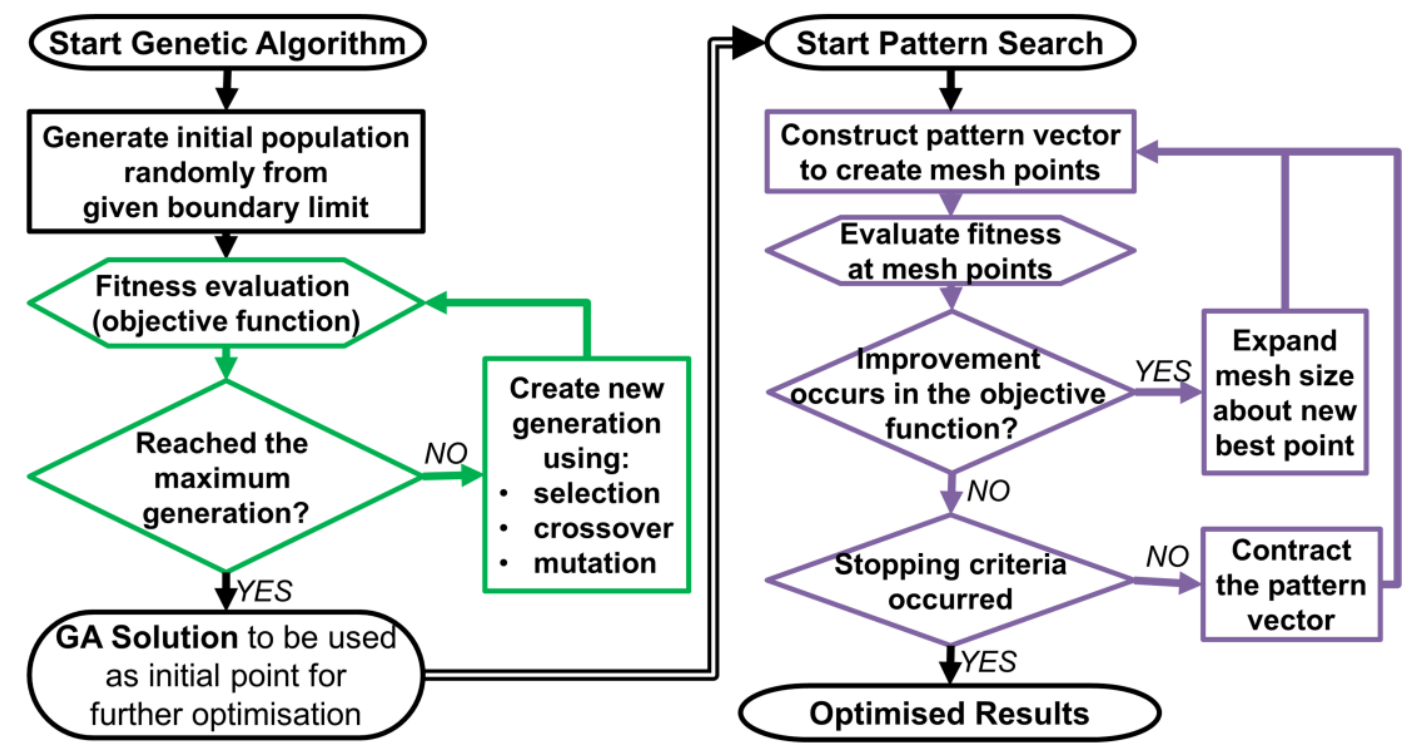

Figure 14. Genetic Algorithm with Pattern Search Method Diagram ${ }^{18}$

\subsection{Application of Optimisation to different Rotors and Turbines}

The optimisation process is first applied to the baseline turbine in Table I and then to turbines with different rotor aspect ratios and turbine ratings.

\subsection{1. $\quad$ Rotor Swept Area Aspect Ratio}

Baseline H-rotor

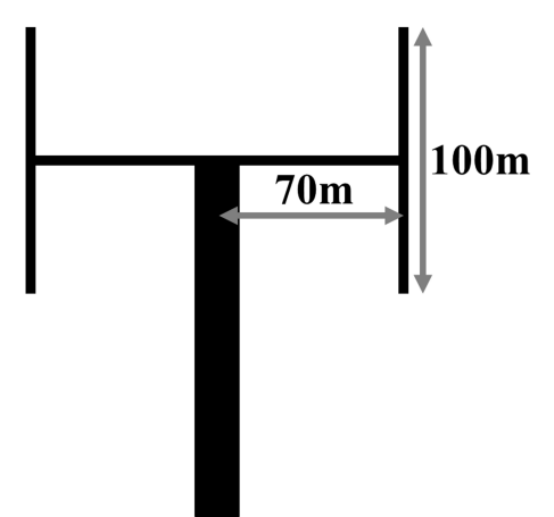

7.7\% Narrower

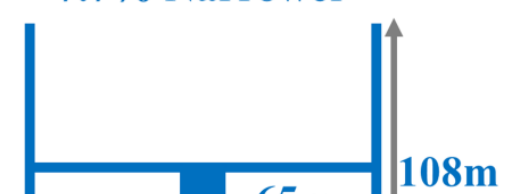

$15.4 \%$ Narrower

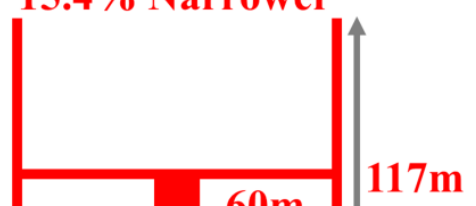

Figure 15. Alternative H-rotor designs

The aspect ratio of the swept area of the turbine rotor blades is defined as the ratio of the diameter of rotation (equal to twice the length rotor arm between tower and blade) divided by the height of the rotor blades. As shown on Figure 15, three potential configurations are tested, the baseline turbine (70m radius, shown in black) and two configurations with reduced aspect ratio (shorter arm radius, with longer blades). These changes lead to two different effects: a change in inertia and a change in tip speed for the same rotational speed. It was decided that two tests per turbine configuration would be carried, one where the moment of inertia was held constant (equal to that of the baseline), and one more realistic run where the moment of inertia decreases in line with the reducing arm length. In all tests the magnitude of the swept area is kept constant at $7000 \mathrm{~m}^{2}$. 


\subsection{2. $\quad$ Turbine Rating}

A further set of tests looked to establish the optimal design of the generator used when the turbine rating varies from 5 to 10 to $20 \mathrm{MW}$. For this test the arm length remained the same whereas the blade length would increase or decrease proportionally with the desired power rating. Also for this test the same generator boundaries for optimisation were used.

\section{RESULTS}

\subsection{Generator Design Optimisation}

The resulting output from the optimisation process is displayed in Table III while a comparison of key outputs from baseline and optimised designs for $\bar{\lambda}=5.3$ (where there is a slight increase in mechanical power with $q$ ) is shown in Figure 16.

Table III shows that the optimisation process produces generator designs with an increased stator radius (which simplistically increases the ratio of energy output to active cost). This is offset by a decrease in stator length resulting in an overall reduction in generator costs. More significantly, these generators have lower losses, resulting in an increase in annual yield and hence a better balance of cost and revenue, as described by $f_{10}$ (Equation (15) where $y=10$ years).

It is important to note that different generators each have their own tailored optimal $q$ strategy. If there is an increase in mechanical torque with $q$ (like in Figure 16) then the optimal strategy $q_{\text {OPT }}(v)$ starts medium to high and reduces as the wind speed increases. For some cases (for example at high tip speed ratios) mechanical torque can be constant with regards to $q$ or even reducing. In these cases the optimal strategy is $q_{\mathrm{OPT}} \sim 0$.

The optimal generator design is larger and more expensive for the higher tip speed ratio.
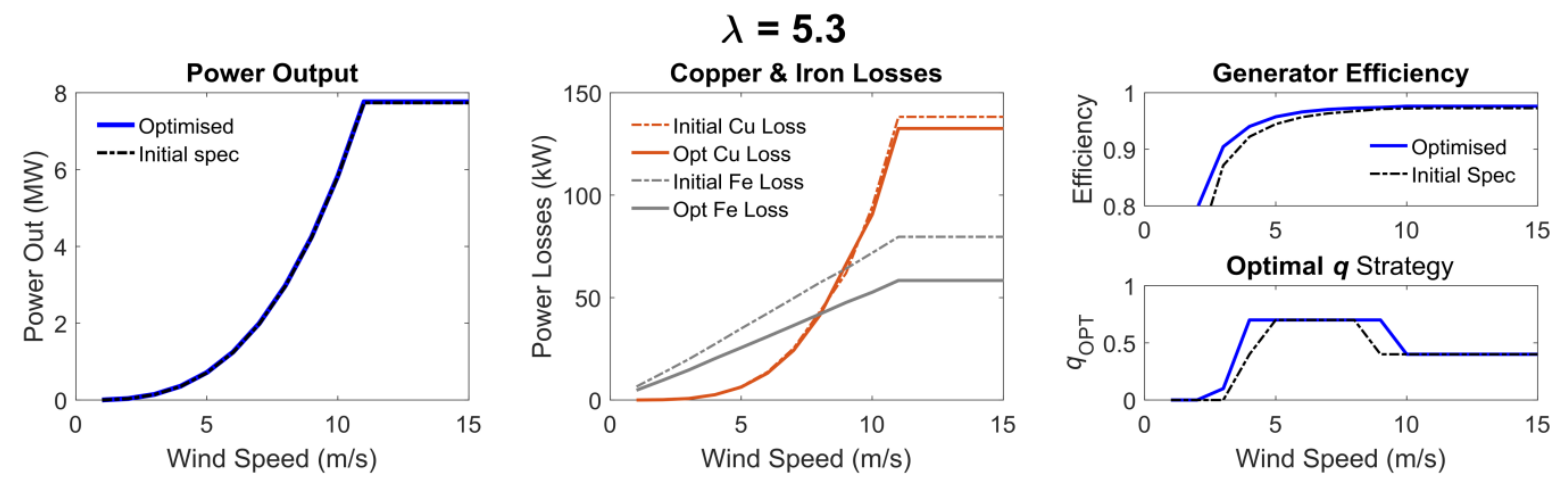

Figure 16. Generator Power Output, Electrical Losses, Generator Efficiency and $\boldsymbol{q}_{\mathbf{O P T}}$ strategy

\subsection{Alternative VAWT Rotor Designs}

\subsubsection{Investigating Rotor Swept Area Aspect Ratio}

The generator output for the different rotor aspect ratios tested is shown in Table IV - in each case the generator is optimised specifically for that turbine design with a separate set of aerodynamic calculations also being carried out for each test.

The relative performance of these designs is also shown visually in Figure 17 where the shorter arm lengths show potential improvement over the baseline specification due to the increase in mechanical power resulting from the increased rotor speed (for the same wind speed and tip speed ratio) which may be worth future investigation. It is also noticeable how varied the $q_{\text {OPT }}$ strategies 
are for the different rotor configurations which further demonstrate how each generator (for each rotor configuration) needs to have its own individually optimised strategy to maximise performance.

(a) Moment of Inertia (J) Fixed
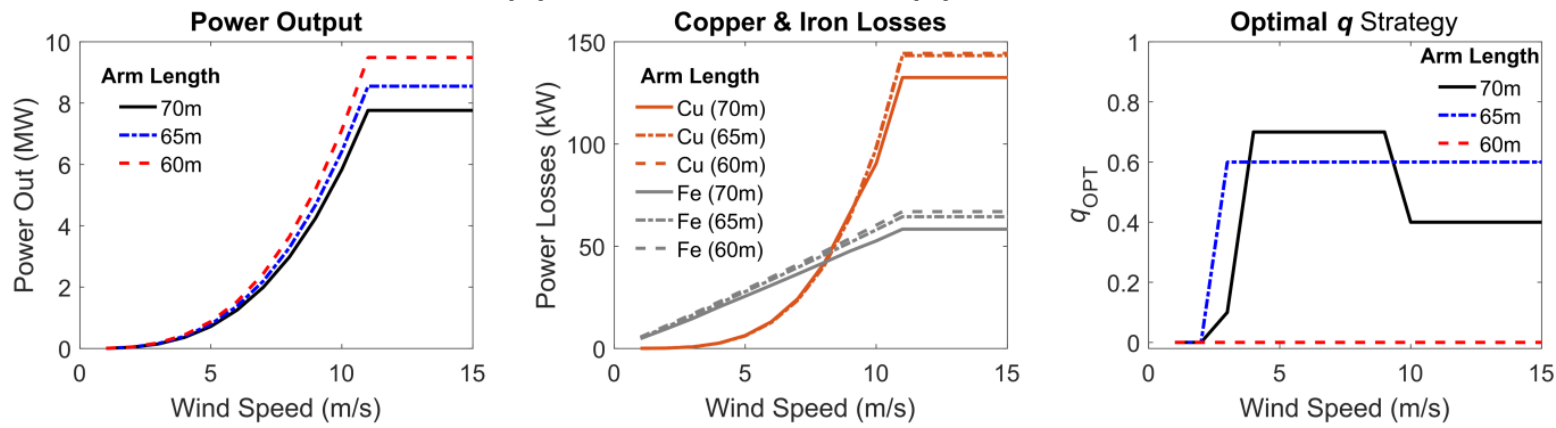

(b) Moment of Inertia (J) Proportional to VAWT Rotor
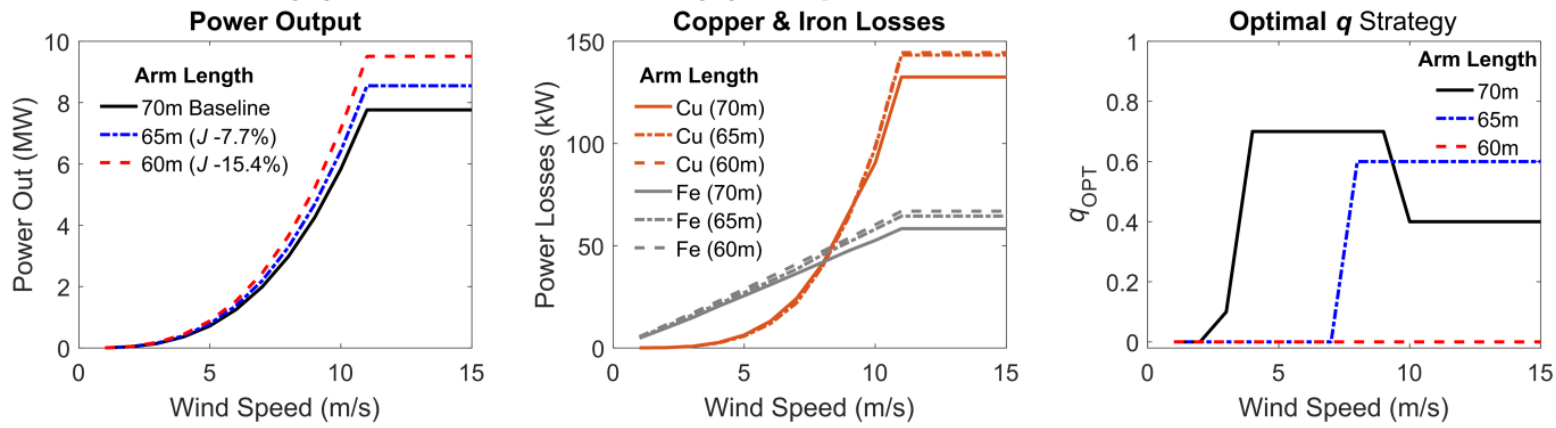

Figure 17. Performance of Generators with Different Rotor Aspect Ratios

\subsubsection{Rescaling to 5MW and 20MW}

The generator specifications and outputs are listed in Table V.
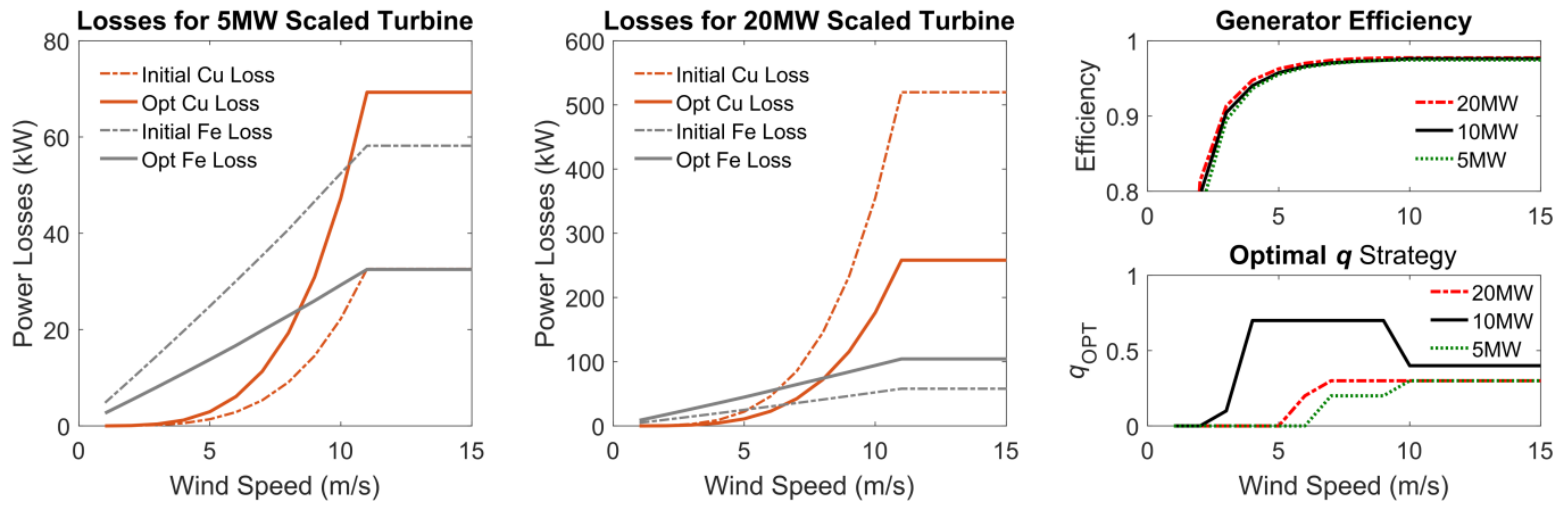

Figure 18. Comparison of Efficiency and Optimal Strategies for 5, 10 \& 20MW turbines

The comparison of the efficiencies of the generators and the corresponding optimal control strategies are shown in Figure 18. The copper and iron losses are also shown in the same figure where the optimised generator is compared to a baseline generator (using the optimised generator parameters from the 10MW generator). In particular, it is noticeable that the optimised $20 \mathrm{MW}$ turbine increases iron losses against the baseline in order to significantly decrease copper losses and thus improve generator efficiency. 


\section{DISCUSSION}

\subsection{Generator Modelling}

The main novelty of this research is the concept of a torque control factor $q$ applied to the electrical torque based on following a proportion of the variation of the mechanical torque as opposed to running at a fixed electrical torque (whether at $\bar{T}$ or otherwise) when wind speed is constant. This work showed how these $q$ strategies affect the generator power losses. While both copper and iron losses increase with $q$ (copper losses increasing with $q^{2}$ ) it may still be advantageous to implement a variable $q$ strategy if the mechanical torque from the rotor blades increases with $q$. It is however unlikely that full torque tracking $(q=1)$ is likely to be optimal due to the large increase in copper losses, especially at higher wind speeds.

In order to ensure a fair comparison of different generator designs, it is important to establish the $q_{\mathrm{OPT}}$ strategy for that generator design. Typically a $q_{\mathrm{OPT}}$ strategy involves running with low or zero electrical torque variation at rated operation. This allows the designer to rate the generator at a lower peak electrical torque and even consider reducing the size of the generator. This is significant as it was assumed at the start of this work that one would have to rate the generator to its peak mechanical torque $(q=1)$ with the corresponding size and cost of the generator, but these results show it is more effective to run at a lower peak electrical torque and potentially reduce the cost of the generator by running a smaller generator in its place.

Since any periodic function (e.g. mechanical torque variation of a VAWT with azimuth angle) can be represented by a Fourier series of sine waves, in practice the torque factor $q$ is applied to each harmonic of the mechanical torque (except the DC offset) proportionally to control the variation in electrical torque. As the mechanical torque trace of a VAWT is dominated by the mean torque $(\bar{T})$ and the $b^{\text {th }}$ harmonic where $b$ is equal to the number of blades (e.g. for a 2-bladed VAWT, the $2^{\text {nd }}$ harmonic), the generator losses can be approximated by simply considering the losses associated with the mean torque and the $b^{\text {th }}$ harmonic. However, when calculating the peak electrical torque, all torque harmonics must be considered.

With a three bladed VAWT the reduced inherent mechanical torque variation would reduce the potential gains from these $q$ strategies compared with two bladed VAWTs. However the effect would still be worth factoring in to the overall Cost of Energy modelling of three bladed VAWTs.

\subsection{Aerodynamic Modelling}

The mechanical power output from section 3.3 shows an increase in mean mechanical power with tip speed. The angle of attack graph and the corresponding $\overline{C_{\mathrm{p}}}-\bar{\lambda}$ (Figure 12) show a decrease in maximum angle of attack from $16.5^{\circ}(\lambda=4)$ to $11^{\circ}(\lambda=6)$; since blade performance usually degrades when the angle of attack is larger than $15^{\circ}$, this backs up why the mean mechanical power output increases with tip speed ratio.

When $q$ is small, there is a relatively large variation in speed so the resulting $C_{\mathrm{p}}$ will vary along the $C_{\mathrm{p}}-\lambda$ curve leading to a potentially large variation in power extraction during each revolution. When the $C_{\mathrm{p}}-\lambda$ curve is increasing: if the curve is steeper above $\bar{\lambda}$ than below then an increase in energy extraction is possible. By contrast when $q$ is close to 1 , the speed variation is reduced and thus there is less variation in power output.

In practice a tip speed ratio above 6 is deemed to be very high for a VAWT and would normally result in a decreasing $C_{\mathrm{p}}-\lambda$ curve due to the increase in drag which is not correctly modelled in this simulation (which is why tip speed ratios above 6 have been omitted). However the main focus of this research is on the powertrain response as opposed to the aerodynamics of the VAWT rotor.

This different behaviour in energy extraction of the VAWT rotor at different speeds has to be incorporated into the overall optimisation loops and therefore the choice of mean tip speed ratio 
depends on running multiple separate optimisations for different mean tip speed ratios and comparing the objective function value of each optimisation result against each other, thereby influencing the optimal powertrain design and operational strategy.

\subsection{Turbine Configuration}

The last set of results look into how changes to the VAWT design affect the powertrain after optimisation. Changing the rotor swept area aspect ratio varied both the aerodynamics and moment of inertia. These results suggest a potential power improvement by narrowing the H-rotor. Note that for a wider $\mathrm{H}$-rotor the rotational speed would drop to the point where the blades are aerodynamically inefficient. Finally, rescaling the generator for 5MW and 20MW resulted in similar "Profit" per MW (although the 20MW turbine was slightly better in this case).

\section{CONCLUSION}

This research shows that the control of electrical torque variation can have an impact on the performance of a commercial scale Vertical Axis Wind Turbine. The selection of $q$ strategy can be important to both the operation and design of the powertrain, as well as influencing the VAWT's wider design. The fixed torque strategy provides good overall turbine performance, while torque tracking is less efficient in terms of losses and generator loading. It may be possible to improve turbine performance by allowing $q$ to vary with respect to wind speed depending on the aerodynamic loading of the rotor but requires robust modelling of such loading patterns. Therefore it is worth integrating this torque variance modelling into analysis of the VAWT powertrain.

The optimisation of powertrain design and operation is best considered as two optimisation procedures (one inside the other) with the aim to reduce the cost of energy contribution of the powertrain. One consequence of this approach is that the powertrain can be rated for a power output only slightly larger than the mean power output at rated operation (as opposed to the much larger peak power output corresponding to the peak mechanical torque of the VAWT rotor). This allows for a smaller and cheaper generator to be used.

The continuation of this research could involve modelling the effect of electrical torque cycling on other aspects that contribute to the cost of energy of a VAWT, e.g. how current cycling affects the failure rate (from fatigue) of components such as the power converters, and how in turn this might affect the operation and maintenance costs of the VAWT.

Generally speaking, a periodic signal (such as torque and it variation with azimuth angle) can be described by its magnitude and its phase. This research has considered the control of the magnitude; further work should investigate varying both the magnitude and phase of the electrical torque (as illustrated in Figure 19). This may have some positive benefits in terms of energy capture. 


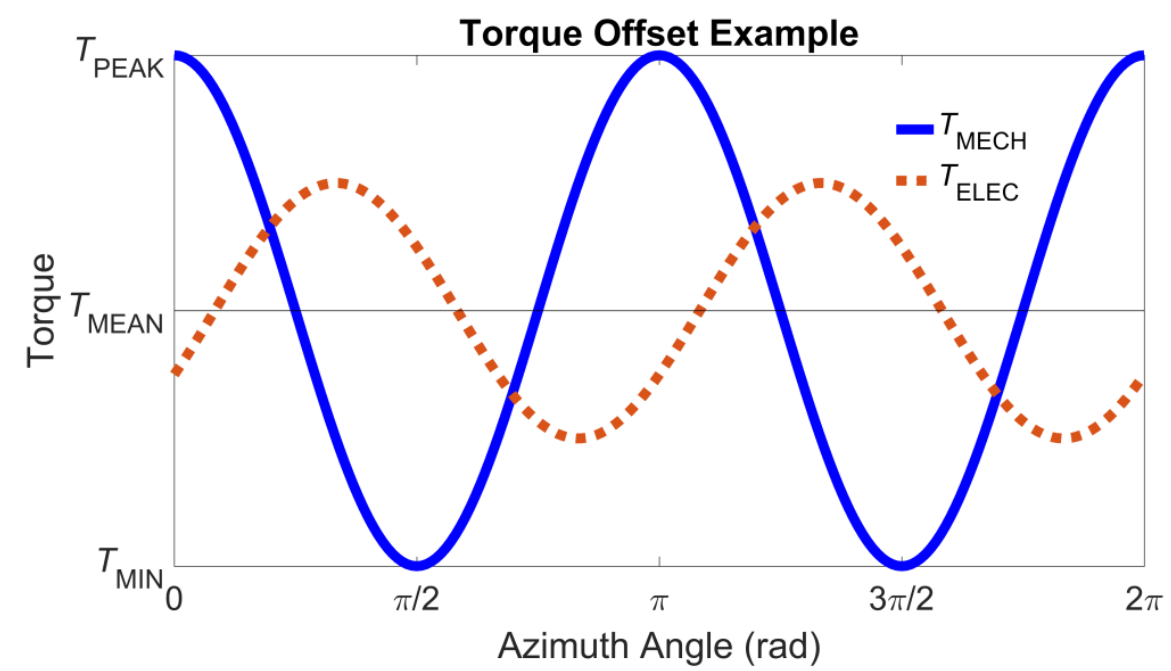

Figure 19. Example of offset between Mechanical and Electrical Torque

\section{REFERENCES}

1. Sutherland HJ, Berg DE, Ashwill TD. A Retrospective of VAWT Technology.; 2012. doi:10.2172/1035336.

2. Shires A. Design optimisation of an offshore vertical axis wind turbine. Energy. 2013;166(EN1):7-18. doi:10.1680/ener.12.00007.

3. Parneix N, Fuchs R, Deglaire P. Efficiency Improvement of Vertical-Axis Wind Turbines with Counter-Rotating Lay-out. In: WindEurope Summit. Hamburg; 2016. https://windeurope.org/summit2016/conference/submit-an-abstract/pdf/12194433729.pdf. Accessed August 8, 2017.

4. VertAx Wind Ltd. Vertical Axis Wind Turbines. http://vertaxwind.com/. Published 2009. Accessed October 14, 2015.

5. Dodd J. First 2MW Vertiwind vertical-axis prototype built. Wind Power Monthly. http://www.windpowermonthly.com/article/1305428/first-2mw-vertiwind-vertical-axisprototype-built. Published 2014. Accessed October 14, 2015.

6. SeaTwirl. Test Lab Lysekil, SeaTwirl S1. http://seatwirl.com/technology/prototype-lysekil. Published 2015. Accessed December 19, 2016.

7. Sandia National Laboratories. Innovative Offshore Vertical-Axis Wind Turbine Rotors Sandia Energy. http://energy.sandia.gov/energy/renewable-energy/wind-power/offshorewind/innovative-offshore-vertical-axis-wind-turbine-rotors/. Accessed December 19, 2016.

8. Siemens Mechanical Drives - Power train optimisation for vertical axis wind turbines. Design Products \& Applications. http://www.dpaonthenet.net/article/106769/Power-trainoptimisation-for-vertical-axis-wind-turbines.aspx. Published 2015. Accessed July 21, 2017.

9. Scheurich F, Fletcher TM, Brown RE. Effect of Blade Geometry on the Aerodynamic Loads Produced by Vertical-Axis Wind Turbines. Proceedings of the Institution of Mechanical Engineers, Part A: Journal of Power and Energy 2011;225(3):327-341. doi:10.1177/2041296710394248.

10. Polinder H, Van Der Pijl FF a, De Vilder GJ, Tavner PJ. Comparison of direct-drive and geared generator concepts for wind turbines. IEEE Trans Energy Convers. 2006;21(3):725733. doi:10.1109/TEC.2006.875476.

11. Argent M, McDonald AS. Vertical Axis Wind Turbine Case Study: Cost and Losses Associated with Variable Torque and Variable Speed Strategies. In: 5th IET International Conference on Renewable Power Generation (RPG) 2016. London: Institution of 
Engineering and Technology; :68 (6 .)-68 (6 .). doi:10.1049/cp.2016.0589.

12. Argent M, Mcdonald A, Leithead B. Vertical Axis Wind Turbines: Minimising Generator Losses by Torque Control. In: 12th European Acadameny of Wind Energy PhD Seminar on Wind Energy in Europe. Lyngby, Denmark: European Academy of Wind Energy. http://strathprints.strath.ac.uk/id/eprint/57649.

13. Soraghan CE. Aerodynamic Modelling and Control of Vertical Axis Wind Turbines. $\begin{array}{llll}\text { University of } & \text { Strathclyde, } & \text { Glasgow } & \text { (Thesis). }\end{array}$ http://ethos.bl.uk/OrderDetails.do?uin=uk.bl.ethos.605986.

14. Ferreira CS. The near wake of the VAWT 2D and 3D views of the VAWT aerodynamics. TU Delft (Thesis). 2009. uuid:ff6eaf63-ac57-492e-a680-c7a50cf5c1cf.

15. Katz J, Plotkin A. Low Speed Aerodynamics. 2nd ed. Cambridge: Cambridge University Press; 2001. doi:10.1017/CBO9780511810329.

16. Zanon A, Giannattasio P, Simão Ferreira CJ. Wake modelling of a VAWT in dynamic stall: impact on the prediction of flow and induction fields. Wind Energy. 2015;18(11):1855-1874. doi:10.1002/we.1793.

17. Soraghan CE, Jamieson P, Leithead PWE. Influence of Lift to Drag Ratio on Optimal Aerodynamic Performance of Straight Blade Vertical Axis Wind Turbines. In: EWEA Annual Wind Energy Event 2013. Vienna, Austria: European Wind Energy Association; 2013. http://strathprints.strath.ac.uk/id/eprint/59292.

18. McDonald A, Bhuiyan NA. On the Optimization of Generators for Offshore Direct Drive Wind Turbines. IEEE Trans Energy Convers. 2017;32(1):348-358. doi:10.1109/TEC.2016.2624219.

19. Hart K, McDonald A, Polinder H, Corr EJ, Carroll J. Improved cost energy comparison of permanent magnet generators for large offshore wind turbines. In: European Wind Energy Association 2014 Annual Conference. Barcelona, Spain: European Wind Energy Association; 2014. http://strathprints.strath.ac.uk/id/eprint/48099.

20. University of Sheffield. Genetic Algorithms Toolbox for Use in MATLAB. http://codem.group.shef.ac.uk/index.php/ga-toolbox. Accessed November 10, 2015.

21. Mathworks. MATLAB Global Optimization Toolbox User's Guide. http://www.mathworks.com/help/pdf_doc/gads/gads_tb.pdf. Accessed December 5, 2015.

22. Giles AD. Wind Panel Manual: Modelling the Aerodynamics of Wind Turbines. October 2017. https://strathprints.strath.ac.uk/63598. Accessed May 1, 2018. 
Table I. Potential VAWT Design and Powertrain Design Options

\begin{tabular}{lccc}
\hline System Design Variable & \multicolumn{3}{c}{ Potential Options } \\
\hline Power / Size of Turbine & 5MW & $\mathbf{1 0 M W}$ & $20 \mathrm{MW}$ \\
VAWT Rotor Design & H-rotor & V-rotor & Darrieus \\
Number of Blades & $\mathbf{2}$ & 3 & $\geq 4$ \\
Swept Area Aspect Ratio & Narrow & Medium & Wide \\
Gearing & Direct Drive & Single Stage & Multiple Stage \\
Generator Type & PMG & DFIG & Other \\
Number of Generators & One & Multiple & - \\
\hline
\end{tabular}

Table shows the potential range of some VAWT design parameters. For this paper, the baseline values are in bold, with variables in grey being investigated as design alternatives in section 5.2. 
Table II. Generator Optimisation Variables

\begin{tabular}{cccc}
\hline Parameter & Lower Bound & Default Value & Upper Bound \\
\hline Stack Length $(\mathrm{m})$ & 0.5 & 1.5 & 8.0 \\
Stator Radius (m) & 2.0 & 7.5 & 8.0 \\
Number of Pole Pairs & 50 & 150 & 480 \\
Magnet Width & $60 \%$ & $70 \%$ & $100 \%$ \\
$\quad$ Pole Pitch & 15 & 40 & 50 \\
Magnet Height (mm) & 15 & 20 & 25 \\
Stator Tooth Width (mm) & 60 & 80 & 80 \\
Stator Tooth Height (mm) & & & \\
\hline
\end{tabular}


Table III. Generator Specifications and Outputs before and after Optimisation

\begin{tabular}{ccc}
\hline Mean Tip Speed Ratio & \multicolumn{2}{c}{$\bar{\lambda}=5.3$} \\
Generator Optimisation Status & Initial Spec & Optimised \\
\hline Stator Length $\times$ Radius $(\mathrm{m})$ & $1.5 \times 7.5$ & $1.30 \times 8.0$ \\
Pole Pairs & 150 & 153 \\
Magnet Width Ratio & $70 \%$ & $60 \%$ \\
Magnet Width $\times$ Height $(\mathrm{mm})$ & $100 \times 40.0$ & $98.4 \times 44.1$ \\
Stator Tooth Width $\times$ Height $(\mathrm{mm})$ & $20.0 \times 80$ & $17.6 \times 80$ \\
\hline \multicolumn{3}{c}{ ENERGY OUTPUT \& LOSSES } \\
\hline Energy Yield $(\mathrm{GWh})$ & 39.26 & 39.43 \\
Copper Loss $(\mathrm{GWh})$ & 0.657 & 0.636 \\
Iron Loss $(\mathrm{GWh})$ & 0.527 & 0.386 \\
Generator Active Cost $(\mathrm{kEuro})$ & 843.7 & 770.2 \\
$\boldsymbol{f}_{\mathbf{1 0}: \text { 10 year “Profit" }(\text { MEuro) }}$ & $\mathbf{7 3 . 7 6}$ & $\mathbf{7 4 . 1 5}$ \\
\hline
\end{tabular}


Table IV. Generator Output for varying Rotor Aspect Ratios

\begin{tabular}{|c|c|c|c|c|c|}
\hline Rotor Swept Area Aspect Ratio & $\underline{\text { Baseline }}$ & \multicolumn{2}{|c|}{ 7.7\% narrower } & \multicolumn{2}{|c|}{$\underline{15 \% \text { narrower }}$} \\
\hline Rotor Diameter : Blade Length & $2 \times 70 / 100=1.4$ & \multicolumn{2}{|c|}{$2 \times 65 / 107.7=1.207$} & \multicolumn{2}{|c|}{$2 \times 60 / 116.7=1.028$} \\
\hline Moment of Inertia $\left(10^{6} \mathrm{~kg} \mathrm{~m}^{2}\right)$ & 105 & 105 & 97.5 & 105 & 90 \\
\hline Stator Length $\times$ Radius $(\mathrm{m})$ & $1.30 \times 8.0$ & $1.34 \times 8.0$ & $1.33 \times 8.0$ & $1.32 \times 8.0$ & $1.32 \times 8.0$ \\
\hline Pole Pairs & 153 & 149 & 150 & 147 & 148 \\
\hline Magnet Width Ratio & $60 \%$ & $60 \%$ & $60 \%$ & $60 \%$ & $60 \%$ \\
\hline Magnet Width $\times$ Height $(\mathrm{mm})$ & $98.4 \times 44.1$ & $101 \times 43.6$ & $100 \times 43.7$ & $102 \times 42.9$ & $102 \times 42.9$ \\
\hline Stator Tooth Width $\times$ Height $(\mathrm{mm})$ & $17.6 \times 80$ & $23.7 \times 80$ & $17.5 \times 80$ & $22.5 \times 80$ & $15.8 \times 80$ \\
\hline \multicolumn{6}{|c|}{ ENERGY OUTPUT \& LOSSES } \\
\hline Energy Yield (GWh) & 39.43 & 43.43 & 43.52 & 48.19 & 48.30 \\
\hline Copper Loss (GWh) & 0.636 & 0.674 & 0.673 & 0.680 & 0.680 \\
\hline Iron Loss (GWh) & 0.386 & 0.424 & 0.422 & 0.441 & 0.440 \\
\hline Generator Active Cost (kEuro) & 770.2 & 787.6 & 785.8 & 774.6 & 774.4 \\
\hline$f_{10}: 10$ year "Profit" (MEuro) & 74.15 & 81.73 & 81.72 & 90.79 & 91.00 \\
\hline
\end{tabular}


Table V. Generator Outputs for 5MW and 20MW rescaled turbines

\begin{tabular}{|c|c|c|c|}
\hline Turbine Power Output & Downscaled 5MW & Baseline 10MW & Upscaled 20MW \\
\hline Rotor Diameter : Blade Length & $2 \times 70 / 50=2.8$ & $2 \times 70 / 100=1.4$ & $2 \times 70 / 200=0.7$ \\
\hline Moment of Inertia $\left(10^{6} \mathrm{~kg} \mathrm{~m}^{2}\right)$ & 52.5 & 105 & 210 \\
\hline Stack Length $\times$ Radius $(\mathrm{m})$ & $0.75 \times 8.0$ & $1.30 \times 8.0$ & $2.27 \times 8.0$ \\
\hline Pole Pairs & 176 & 153 & 137 \\
\hline Magnet Width Ratio & $60 \%$ & $60 \%$ & $60 \%$ \\
\hline Magnet Width $\times$ Height $(\mathrm{mm})$ & $85.5 \times 37.9$ & $98.4 \times 44.1$ & $110 \times 49.6$ \\
\hline Stator Tooth Width $\times$ Height $(\mathrm{mm})$ & $19.4 \times 80$ & $17.6 \times 80$ & $23.7 \times 80$ \\
\hline \multicolumn{4}{|c|}{ ENERGY OUTPUT \& LOSSES } \\
\hline Energy Yield (GWh) & 19.65 & 39.43 & 78.85 \\
\hline Copper Loss (GWh) & 0.326 & 0.636 & 1.215 \\
\hline Iron Loss (GWh) & 0.213 & 0.386 & 0.688 \\
\hline Generator Active Cost (kEuro) & 434.4 & 770.2 & 1382 \\
\hline$f_{10}: 10$ year "Profit" (MEuro) & 36.90 & 74.15 & 148.4 \\
\hline "Profit" per MW (MEuro) & 7.380 & 7.415 & 7.422 \\
\hline
\end{tabular}

